\title{
ENRICHED RIEMANN SPHERE, MORSE STABILITY AND EQUI-SINGULARITY IN $\mathcal{O}_{2}$
}

\author{
TZEE-CHAR KUO AND LAURENTIU PAUNESCU
}

\begin{abstract}
The Enriched Riemann Sphere $\mathbb{C} P_{*}^{1}$ is $\mathbb{C} P^{1}$ plus a set of infinitesimals, having the Newton-Puiseux field $\mathbb{F}$ as coordinates. Complex Analysis is extended to the $\mathbb{F}$-Analysis (Newton-Puiseux Analysis). The classical Morse Stability Theorem is also extended; the stability idea is used to formulate an equi-singular deformation theorem in $\mathbb{C}\{x, y\}\left(=\mathcal{O}_{2}\right)$.
\end{abstract}

A general principle we believe in is that the study of convergent power series in $n+1$ variables is Global Analysis of polynomials in $n$ variables.

In this paper this is illustrated in the case $n=1$. Loosely speaking, the classical Morse Stability Theorem, properly reformulated in \$10, and the stability notion are "transplanted" into Algebraic Curves, then applied to the classification problem of singularities.

In $\mathbb{1}_{1}$, the Riemann sphere $\mathbb{C} P^{1}$ is "enriched" to $\mathbb{C} P_{*}^{1}$ with "infinitesimals", which are irreducible curve-germs, and $\mathbb{C}$ to $\mathbb{C}_{*}$. The Newton-Puiseux field $\mathbb{F}$ of convergent fractional power series is used as coordinates, in terms of which several structures are defined.

In $\oint_{2}$, the Cauchy Integral Theorem, Taylor expansions, critical points, stability, etc., are generalized to $\mathbb{F}$, as is the classical Morse Stability Theorem.

The notion of Morse stability for polynomials over $\mathbb{F}$ suggests a stronger definition for "equi-singular deformation" in $\mathbb{C}^{2}$ (Compare [25]).

For example, in contemporary Algebraic Geometry, both deformations

$$
Q(x, y, t):=x^{4}-t^{2} x^{2} y^{2}+y^{4}, \quad P(x, y, t):=x^{3}-y^{4}-3 t^{2} x y^{2 d}, d \geq 2,
$$

are regarded as equi-singular: the zero sets are topologically trivial (Milnor $\mu$-constant).

As we shall see, however, $Q$ is not equi-singular from our viewpoint. The hypothesis of the Equi-singular Deformation Theorem in $\$ 3$ is not satisfied. The associated family $\xi^{4}-t^{2} \xi^{2}+1$ is not Morse stable $(\xi=0$ splits into three critical points when $t \neq 0)$.

On the other hand, $P$, the Pham family $([20])$, is equi-singular in the sense of Definition 2.7, although the "polar" $P_{x}$ splits into $x \pm t y^{d}$. This is explained in Attention8.2 and Example 2.8, The associated family $\xi^{3}-1$, being independent of $t$, is obviously Morse stable in the sense of Definition 5.4. Our Equi-singular Deformation Theorem applies.

Date: November 5, 2018.

2000 Mathematics Subject Classification. Primary 14HXX, 32SXX, 58K60, Secondary 58K40.

Key words and phrases. Fractional power series, Curve-germs, Infinitesimals, Newton-Puiseux analysis, Morse stability, Classification of singularities. 
When does a given family $F(x, y, t)$, like $Q, P$ above, admit a trivialization, and of what kind? An answer is given in the Equi-singular Deformation Theorem in $§ 3$, using the notion of Morse stability. (Similar results over $\mathbb{R}$ were announced in [15].)

The Truncation Theorem at the end of $\$ 3$ asserts that $f(x, y)$ can be equi-singularly deformed into its "Puiseux root truncation" $\hat{f}_{\text {root }}(x, y)$. (We do not assume 0 is an isolated singularity.) This theorem is closely related to results on sufficiency of jets, like Morse Lemma, [1], 2], 5], 8], [1], [12], [18], 19], etc.. Compare also the classical book [9].

\section{The Enriched Riemann Sphere}

Take a holomorphic map-germ $\mathcal{A}:(\mathbb{C}, 0) \rightarrow\left(\mathbb{C}^{2}, 0\right), \mathcal{A}(z) \neq 0$ if $z \neq 0$. The image setgerm, $\operatorname{Im}(\mathcal{A})$, or the geometric locus of $\mathcal{A}$, has a well-defined tangent line $T(\mathcal{A})$ at 0 . We call $\operatorname{Im}(\mathcal{A})$ an infinitesimal at $T(\mathcal{A}) \in \mathbb{C} P^{1}$. The set of infinitesimals is denoted by $\mathbb{C} P_{*}^{1}$.

The geometric locus of $z \mapsto(a z, b z)$ is identified with $[a: b] \in \mathbb{C} P^{1}$, hence $\mathbb{C} P^{1} \subset \mathbb{C} P_{*}^{1}$.

For instance, the curve-germ $x^{2}-y^{3}=0$, being the geometric locus of $z \mapsto\left(z^{3}, z^{2}\right)$, is an infinitesimal at $[0: 1]$. It is "closer" to $[0: 1]$ than any $[a: 1]$ is, $a \neq 0$, in the sense that its contact order (defined below) with $x=0$ is higher than that between $x=a y$ and $x=0$.

As in Projective Geometry, $\mathbb{C} P_{*}^{1}$ is the union $\mathbb{C P}_{*}^{1}=\mathbb{C}_{*} \cup \mathbb{C}_{*}^{\prime}$, where

$$
\mathbb{C}_{*}:=\{\operatorname{Im}(\mathcal{A}) \mid T(\mathcal{A}) \neq[1: 0]\}, \quad \mathbb{C}_{*}^{\prime}:=\{\operatorname{Im}(\mathcal{A}) \mid T(\mathcal{A}) \neq[0: 1]\} .
$$

The classical Newton-Puiseux Theorem asserts that the field $\mathbb{F}$ of convergent fractional power series in an indeterminate $y$ is algebraically closed. ([6], [10], [21], 22], [23].)

Recall that a non-zero element of $\mathbb{F}$ is a (finite or infinite) convergent series

$$
\alpha: \alpha(y)=a_{0} y^{n_{0} / N}+\cdots+a_{i} y^{n_{i} / N}+\cdots, \quad n_{0}<n_{1}<\cdots,
$$

where $N \in \mathbb{Z}^{+}, n_{i} \in \mathbb{Z}, 0 \neq a_{i} \in \mathbb{C}$. The order of $\alpha$ is $O_{y}(\alpha):=n_{0} / N, O_{y}(0):=+\infty$.

We can assume $G C D\left(N, n_{0}, n_{1}, \ldots\right)=1$. In this paper we call $m_{\text {puis }}(\alpha):=N$ the Puiseux multiplicity of $\alpha$ (for clarity). The conjugates of $\alpha$ are

$$
\alpha_{\text {conj }}^{(k)}(y):=\sum a_{i} \theta^{k n_{i}} y^{n_{i} / N}, \quad 0 \leq k \leq N-1, \quad \theta:=e^{2 \pi \sqrt{-1} / N} .
$$

The following $\mathbb{D}$ is an integral domain with quotient field $\mathbb{F}$ and ideals $\mathbb{M}, \mathbb{M}_{1}$ :

$$
\mathbb{D}:=\left\{\alpha \in \mathbb{F} \mid O_{y}(\alpha) \geq 0\right\}, \mathbb{M}:=\left\{\alpha \mid O_{y}(\alpha)>0\right\}, \mathbb{M}_{1}:=\left\{\alpha \mid O_{y}(\alpha) \geq 1\right\} .
$$

Define $|\alpha|:=\sum 2^{-n_{i} / N}\left|a_{i}\right|\left(1+\left|a_{i}\right|\right)^{-1}$. Then $d(\alpha, \beta):=|\alpha-\beta|$ is a metric on $\mathbb{D}$. If we fix $N$, then $\lim _{m \rightarrow \infty} \sum a_{i}(m) y^{n_{i} / N}=0$ iff each $a_{i}(m) \rightarrow 0$ (point-wise convergence).

Given $\alpha \in \mathbb{M}_{1}$, let $\mathcal{A}(z):=\left(\alpha\left(z^{N}\right), z^{N}\right)$. We then define $\alpha_{*}:=\pi_{*}(\alpha):=\operatorname{Im}(\mathcal{A})$, and use $\pi_{*}: \mathbb{M}_{1} \rightarrow \mathbb{C}_{*}$, a many-to-one surjective mapping, as a coordinate system on $\mathbb{C}_{*}$.

A coordinate system on $\mathbb{C}_{*}^{\prime}$ is $\pi_{*}^{\prime}: \mathbb{M}_{1} \rightarrow \mathbb{C}_{*}^{\prime}, \alpha_{*}:=\pi_{*}^{\prime}(\alpha):=\operatorname{Im}(\mathcal{A}), \mathcal{A}(z):=\left(z^{N}, \alpha\left(z^{N}\right)\right)$.

We furnish $\mathbb{C}_{*}\left(\right.$ resp. $\left.\mathbb{C}_{*}^{\prime}\right)$ with the quotient topology of $\pi_{*}$ (resp. $\left.\pi_{*}^{\prime}\right)$. As for the transition function in the overlap $\mathbb{C}_{*} \cap \mathbb{C}_{*}^{\prime}$, take $x=\alpha(y), n_{0} / N=1$, we then "solve $y$ in terms of $x$ ", obtaining $y=\beta(x):=b_{0} x+b_{1} x^{n_{1}^{\prime} / N^{\prime}}+\cdots$, where $a_{0} b_{0}=1$, each $b_{i}$ is a polynomial in finitely many of $\left(\sqrt[N]{a_{0}}\right)^{-1}, a_{1} / a_{0}, a_{2} / a_{0}, \ldots$. Hence the topologies coincide in $\mathbb{C}_{*} \cap \mathbb{C}_{*}^{\prime}$.

The quotient topology on $\mathbb{C} P_{*}^{1}$ is well-defined. 
ENRICHED RIEMANN SPHERE, MORSE STABILITY AND EQUI-SINGULARITY IN $\mathcal{O}_{2}$

Let $X, Y \subset \mathbb{R}^{n}$ be germs of sub-analytic sets at $0, X \cap Y=\{0\}, X \neq\{0\} \neq Y$. Define the contact order $\mathcal{C}_{\text {ord }}(X, Y)$ to be the smallest number $L$ (the Lojasiewicz exponent) such that $d(x, y) \geq a\|(x, y)\|^{L}$, where $x \in X, y \in Y,\|x\|=\|y\|, a>0$ constant ([4]).

In particular, for $\alpha_{*}, \beta_{*} \in \mathbb{C} P_{*}^{1}, \mathcal{C}_{\text {ord }}\left(\alpha_{*}, \beta_{*}\right)$ is defined; $\mathcal{C}_{\text {ord }}\left(\alpha_{*}, \alpha_{*}\right):=\infty$. Thus, in $\mathbb{C}_{*}$,

$$
\mathcal{C}_{\text {ord }}\left(\alpha_{*}, \beta_{*}\right)=\max _{j}\left\{O_{y}\left(\alpha-\beta_{\text {conj }}^{(j)}\right)\right\}=\max _{k, j}\left\{O_{y}\left(\alpha_{c o n j}^{(k)}-\beta_{c o n j}^{(j)}\right)\right\} .
$$

This is the contact order structure on $\mathbb{C} P_{*}^{1}$.

The Puiseux (characteristic) pairs of $\alpha([24])$, which describes the iterated torus knot of the curve-germ $\alpha_{*}$, is denoted by $\chi_{\text {puis }}(\alpha)$ or $\chi_{\text {puis }}\left(\alpha_{*}\right)$.

The Enriched Riemann Sphere is $\mathbb{C P}_{*}^{1}$ furnished with the above structures, $\mathbb{C}_{*}$ is the enriched complex plane. (The Riemann-Zariski surface ([7], p.272) is much larger than $\mathbb{C} P_{*}^{1}$. For example, $x=y^{\sqrt{2}}$ defines a point in the former, but not in the latter.)

Convention 1.1. Throughout this paper, $\epsilon>0$ is a sufficiently small constant,

$$
I_{\mathbb{R}}:=\{t \in \mathbb{R}|| t \mid<\epsilon\}, \quad I_{\mathbb{C}}:=\{t \in \mathbb{C}|| t \mid<\epsilon\}, \quad I_{\mathbb{F}}:=\{t \in \mathbb{D}|| t \mid<\epsilon\} .
$$

We say $\varphi(w)$ is real analytic, $w=u+\sqrt{-1} v \in \mathbb{C}$, if it is so as a function of $(u, v) \in \mathbb{R}^{2}$. By "+..." we mean "plus higher order terms".

\section{The $\mathbb{F}$-Analysis (Newton-Puiseux Analysis)}

Given $U \subset \mathbb{D}$, open, and $\phi: U \rightarrow \mathbb{F}$. We say $\phi$ is Puiseux-Lojasiewicz bounded if every $\alpha \in U$ has a neighbourhood $\mathcal{N}(\alpha)$ with constants $K(\alpha), L(\alpha)>0$, such that

$$
m_{\text {puis }}(\phi(\xi)) \leq K(\alpha) m_{\text {puis }}(\xi), \quad O_{y}(\phi(\xi)) \geq-L(\alpha), \quad \xi \in \mathcal{N}(\alpha) .
$$

In this paper we only study functions which are Puiseux-Lojasiewicz bounded.

Definition 2.1. We say $\phi$ is differentiable at $\alpha \in U$, with derivative $\phi^{\prime}(\alpha) \in \mathbb{F}$, if

$$
\phi^{\prime}(\alpha)=\lim [\phi(\alpha+\delta)-\phi(\alpha)] / \delta \text { as } \delta \rightarrow 0(\delta \in \mathbb{D}) .
$$

If $\phi^{\prime}(\gamma)=0, \gamma$ is a critical point, with multiplicity

$$
m_{\text {crit }}(\gamma):=\max \left\{k \mid \phi^{(i)}(\gamma)=0,1 \leq i \leq k\right\} .
$$

Cauchy's Theorem. If $\phi^{\prime}(\alpha)$ exists at every $\alpha \in U$, then all derivatives $\phi^{(k)}(\alpha)$ exist,

$$
\oint_{z \in C} \phi(\mu) d \mu=0, \quad \phi^{(k)}(\alpha)=\frac{k !}{2 \pi \sqrt{-1}} \oint_{z \in C} \frac{\phi(\mu)}{(\mu-\alpha)^{k+1}} d \mu, k \geq 0,
$$

where $\mu:=\alpha+z \delta, \delta \in \mathbb{D}, d \mu:=\delta d z, C$ a sufficiently small contour around $0 \in \mathbb{C}$.

Moreover, $\phi$ is $\mathbb{F}$-analytic in the sense that if $\alpha+z \delta \in U,|z|<r, z \in \mathbb{C}$, then

$$
\phi(\alpha+z \delta)=\phi(\alpha)+\cdots+(1 / k !) \phi^{(k)}(\alpha)(z \delta)^{k}+\cdots,|z|<r,
$$

where $m_{\text {puis }}\left(\phi^{(k)}(\alpha)\right) \leq K(\alpha)$, a constant. 
From now on, we consider a given $\phi: \mathbb{M}_{1} \rightarrow \mathbb{M}_{1}$, which extends to a differentiable function $U \rightarrow \mathbb{D}$. Taking $\alpha, \delta \in \mathbb{M}_{1}, \xi:=z \delta$, we have the "Taylor expansion" of $\phi$ at $\alpha$ :

$$
\phi(\alpha+\xi)=\sum \alpha_{k} \xi^{k}, \quad \xi \in \mathbb{M}_{1}, \quad \alpha_{k}:=(1 / k !) \phi^{(k)}(\alpha) \in \mathbb{D} .
$$

In this paper we always assume $\phi$ is mini-regular in $\xi$, say of order $m$, i.e.,

$$
O_{y}\left(\alpha_{m}\right)=0, \quad O_{y}\left(\alpha_{k}\right)+k \geq m \text { for } 0 \leq k \leq m-1 .
$$

Thus, by the Newton-Puiseux Theorem, $\phi$ has $m$ roots in $\mathbb{M}_{1}$,

$$
Z(\phi):=\left\{\zeta \in \mathbb{M}_{1} \mid \phi(\zeta)=0\right\}:=\left\{\zeta_{1}, \ldots, \zeta_{d}\right\}, \quad m=\sum m_{i},
$$

where $m_{i}:=m\left(\zeta_{i}\right)$ is the multiplicity of $\zeta_{i}$. Of course $\phi$ has $m-1$ critical points in $\mathbb{M}_{1}$.

Definition 2.2. In $\mathbb{M}_{1}$, define $\mu \sim_{\phi} \nu$ iff either $\mu=\nu$ or else

$$
O_{y}(\mu-\nu)>O_{y}\left(\mu-\zeta_{i}\right)=O_{y}\left(\nu-\zeta_{i}\right), \quad 1 \leq i \leq d .
$$

The equivalence class of $\mu$ is denoted by $\mu_{\phi}$. The height of $\mu_{\phi}$ is

$$
h\left(\mu_{\phi}\right):=\max \left\{O_{y}\left(\mu-\zeta_{i}\right) \mid 1 \leq i \leq d\right\} .
$$

The quotient space is $\mathbb{M}_{1, \phi}:=\mathbb{M}_{1} / \sim_{\phi}$, with the contact order structure:

$$
\mathcal{C}_{\text {ord }}\left(\mu_{\phi}, \nu_{\phi}\right):=O_{y}(\mu-\nu) \text { if } \mu_{\phi} \neq \nu_{\phi} ; \mathcal{C}_{\text {ord }}\left(\mu_{\phi}, \mu_{\phi}\right):=\infty .
$$

Let $\mu_{\phi}(y)$ denote $\mu(y)$ with terms $y^{e}$ deleted, $e>h\left(\mu_{\phi}\right) ; \mu_{\phi}(y)$ depends only on $\mu_{\phi} \in \mathbb{M}_{1, \phi}$. We call $\mu_{\phi}(y) \in \mathbb{M}_{1}$ the canonical coordinate of $\mu_{\phi} \in \mathbb{M}_{1, \phi} ; \mu_{\phi}$ and $\mu_{\phi}(y)$ are often identified.

The Puiseux pairs of $\mu_{\phi} \in \mathbb{M}_{1, \phi}$ is $\chi_{\text {puis }}\left(\mu_{\phi}\right):=\chi_{\text {puis }}\left(\mu_{\phi}(y)\right)$.

Example 2.3. . Take $\phi(\xi):=\xi^{2}-2 y^{3}, \mu(y):=y^{3 / 2}+y^{7 / 4}$. Then $h\left(\mu_{\phi}\right)=3 / 2, \mu_{\phi}(y)=y^{3 / 2}$, $\chi_{\text {puis }}(\mu)=\{3 / 2,7 / 4\}, \chi_{\text {puis }}\left(\mu_{\phi}\right)=\{3 / 2\}$. (We also call $\mu_{\phi}(y)$ the $\phi$-truncation of $\mu(y)$.)

The tree-model of $\phi$ defined in [13] is our $\mathbb{M}_{1, \phi}$ without the structures. See $\$ 11$.

Definition 2.4. In $\mathbb{M}_{1, \phi}$ define $\xi_{\phi} \sim_{b a r} \eta_{\phi}$ iff either $\xi_{\phi}=\eta_{\phi}$, or else

$$
h\left(\xi_{\phi}\right)=h\left(\eta_{\phi}\right)=\mathcal{C}_{\text {ord }}\left(\xi_{\phi}, \eta_{\phi}\right) .
$$

An equivalence class is called a bar (as in [13]). The bar space is the quotient

$$
\operatorname{Bsp}\left(\mathbb{M}_{1, \phi}\right):=\mathbb{M}_{1, \phi} / \sim_{b a r} .
$$

The bar containing $\xi_{\phi}$ is denoted by $B\left(\xi_{\phi}\right)$, having height $h\left(B\left(\xi_{\phi}\right)\right):=h\left(\xi_{\phi}\right) ; B_{h}(\phi)$ denotes a bar of height $h$. The Lojasiewicz exponent of $\phi$ at $\xi$, or at $\xi_{\phi}$, or on $B\left(\xi_{\phi}\right)$, is

$$
L_{\phi}(\xi):=L\left(\xi_{\phi}\right):=L\left(B\left(\xi_{\phi}\right)\right):=O_{y}(\phi(\xi)) .
$$

That (2.6) is well-defined is an easy consequence of the following:

$$
\phi(\xi)=\text { unit } \cdot \prod\left(\xi-\zeta_{i}\right)^{m_{i}}, \quad O_{y}(\phi(\xi))=\sum m_{i} O_{y}\left(\xi-\zeta_{i}\right) .
$$

An important special case is $\mathcal{V}:=\mathbb{M}_{1, i d}$ when $\phi=i d: \xi \mapsto \xi$. Here $Z(i d)=\{0\}$.

We call $\mathcal{V}$ the value space, and $0_{\mathcal{V}}:=0_{i d}$ the "zero" element. 
If $\mu(y)=u y^{h}+\cdots, u \neq 0$, then $\mu_{i d}$ is completely determined by the pair $(u, h)$. Hence, if $h<\infty$, there is a unique bar of height $h, B_{h}(i d)=\{(u, h) \mid u \neq 0\}$; this is a copy of $\mathbb{C}-\{0\}$.

For $h=\infty$, we have a singleton $B_{\infty}(i d)=\left\{0_{\mathcal{V}}\right\}$.

Definition 2.5. The valuation function, val, also written as $v_{a l}$ for clarity, is

$$
\operatorname{val}:=\operatorname{val}_{\phi}: \mathbb{M}_{1, \phi} \rightarrow \mathcal{V}, \quad \operatorname{val}\left(\xi_{\phi}\right):=\operatorname{val}_{\phi}\left(\xi_{\phi}\right):=\phi(\xi)_{i d}
$$

If $\phi^{\prime}(\gamma)=0, \gamma \in \gamma_{\phi}, \gamma_{\phi}$ is a critical point of $v a l_{\phi}$. The multiplicity, $m_{c r i t}\left(\gamma_{\phi}\right)$, is the total number of such $\gamma \in \gamma_{\phi}$.

The subspace $C\left(v a l_{\phi}\right)$ of critical points is displayed as

$$
C\left(v_{a l}\right):=\left\{\gamma_{1, \phi}, \ldots, \gamma_{p, \phi}\right\}, \quad \sum m_{c r i t}\left(\gamma_{j, \phi}\right)=m-1 .
$$

Example. Take $\phi(\xi)=\xi^{4}(\xi-y)^{5}, \varepsilon \neq 0, h>1$. Then $\operatorname{val}_{\phi}\left(\varepsilon y^{h}+\cdots\right)=\left(-\varepsilon^{4}, 4 h+5\right)$, $\operatorname{val}_{\phi}((1+\varepsilon) y+\cdots)=\left(\varepsilon^{5}(1+\varepsilon)^{4}, 9\right), \operatorname{val}_{\phi}(0)=\operatorname{val}_{\phi}(y)=0_{\mathcal{V}}$.

Definition 2.6. Let $\phi$ be as in (2.3), (2.4). Take

$$
\Phi(\xi, t):=\sum A_{i}(t) \xi^{i} \in \mathbb{F}\{\xi, t\}, \quad \Phi(\xi, 0)=\phi(\xi) .
$$

We call $\Phi(\xi, t):=\phi_{t}(\xi)$ an $\mathbb{F}$-analytic deformation of $\phi$.

In this paper, we always assume $A_{i}(t) \in \mathbb{D}\{t\}$, and $\Phi$ is mini-regular:

$$
O_{y}\left(A_{i}(t)\right)+i \geq m, \quad 0 \leq i \leq m .
$$

In $\mathbb{M}_{1} \times I_{\mathbb{F}}$, define $(\mu, t) \sim_{\Phi}\left(\nu, t^{\prime}\right)$ iff $t=t^{\prime}, \mu_{\phi_{t}}=\nu_{\phi_{t}}$.

The quotient space is $\mathbb{M}_{1} \times_{\Phi} I_{\mathbb{F}}:=\mathbb{M}_{1} \times I_{\mathbb{F}} / \sim_{\Phi}$, with valuation function

$$
\operatorname{val}_{\Phi}: \mathbb{M}_{1} \times_{\Phi} I_{\mathbb{F}} \rightarrow \mathcal{V}, \quad\left(\xi_{\phi_{t}}, t\right) \mapsto\left[\phi_{t}(\xi)\right]_{i d}
$$

The subspace of critical points is

$$
C\left(v_{a l}\right):=\left\{\left(\gamma_{\phi_{t}}, t\right) \in \mathbb{M}_{1} \times_{\Phi} I_{\mathbb{F}} \mid \gamma_{\phi_{t}} \in C\left(v_{a l} l_{\phi_{t}}\right)\right\} .
$$

The bar space $B \operatorname{sp}\left(\mathbb{M}_{1} \times_{\Phi} I_{\mathbb{F}}\right)$ is similarly defined.

Definition 2.7. We say $\Phi$ is almost Morse stable if there exists a homeomorphism

$$
\tau: C\left(v l_{\phi}\right) \times I_{\mathbb{F}} \rightarrow C\left(v_{a l}\right), \quad\left(\gamma_{\phi}, t\right) \mapsto\left(\tau_{t}\left(\gamma_{\phi}\right), t\right),
$$

where $L\left(\tau_{t}\left(\gamma_{\phi}\right)\right)=L\left(\gamma_{\phi}\right)$.

We say $\Phi$ is Morse stable if, in addition, the following holds.

Given $\gamma_{\phi}, \gamma_{\phi}^{\prime} \in C\left(v a l_{\phi}\right)$. If $B\left(\gamma_{\phi}\right)=B\left(\gamma_{\phi}^{\prime}\right)$ and $v a l_{\phi}\left(\gamma_{\phi}\right)=\operatorname{val}_{\phi}\left(\gamma_{\phi}^{\prime}\right)$, then

$$
\operatorname{val}_{\phi_{t}}\left(\tau_{t}\left(\gamma_{\phi}\right)\right)=\operatorname{val}_{\phi_{t}}\left(\tau_{t}\left(\gamma_{\phi}^{\prime}\right)\right), \quad B\left(\tau_{t}\left(\gamma_{\phi}\right)\right)=B\left(\tau_{t}\left(\gamma_{\phi}^{\prime}\right)\right) \text {. }
$$

Example 2.8. For $P(x, y, t)$ in (0.1) $, \Phi(\xi, t):=\xi^{3}-3 t^{2} y^{2 d} \xi-y^{4}$, val $\phi_{t}$ has a unique critical point $\gamma_{\phi_{t}}=0$ in $\mathbb{M}_{1, \phi_{t}}, m_{\text {crit }}\left(\gamma_{\phi_{t}}\right)=2$. With $\tau_{t}=i d$, $\Phi$ is Morse stable.

Attention: $\phi_{t}$ has two critical points $\xi= \pm t y^{d}$ in $\mathbb{M}_{1}$, but val $\phi_{t}$ has only one in $\mathbb{M}_{1, \phi_{t}}$.

On the other hand, $\Psi(\xi, t):=\xi^{3}+t y^{3}-y^{4}$ is not almost Morse stable. 
Let $\mathcal{V} \times_{L o j} B \operatorname{sp}\left(\mathbb{M}_{1, \phi}\right):=\{(v, B) \mid v \in \mathcal{V}, L(v)=L(B)\} \subset \mathcal{V} \times B \operatorname{sp}\left(\mathbb{M}_{1}, \phi\right)$. We may call this a "Lojasiewicz fiber product". Define $\mathcal{V} \times_{\text {Loj }} B \operatorname{sp}\left(\mathbb{M}_{1} \times_{\Phi} I_{\mathbb{F}}\right)$ similarly.

The Morse Stability Theorem (Over $\mathbb{F}$ ). Suppose $\Phi$ is Morse stable. Then there exist t-level preserving homeomorphisms (deformations) $\mathcal{D}_{\Phi}, \mathcal{D}_{\mathcal{V}}$ such that the diagram

is commutative, where

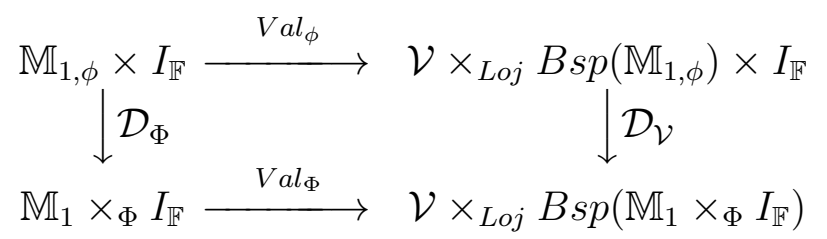

$$
\operatorname{Val}_{\phi}\left(\mu_{\phi}, t\right):=\left(\operatorname{val}_{\phi}\left(\mu_{\phi}\right), B\left(\mu_{\phi}\right), t\right), \operatorname{Val}_{\Phi}\left(\mu_{\phi_{t}}, t\right):=\left(\operatorname{val}_{\phi_{t}}\left(\mu_{\phi_{t}}\right), B\left(\mu_{\phi_{t}}\right), t\right) .
$$

The structures are preserved. That is, if $\mathcal{D}_{\Phi}\left(\mu_{\phi}, t\right):=\left(\mu_{\phi, t}, t\right), \mu_{\phi, t} \in \mathbb{M}_{1, \phi_{t}}$, then

$$
h\left(\mu_{\phi, t}\right)=h\left(\mu_{\phi}\right), \chi_{\text {puis }}\left(\mu_{\phi, t}\right)=\chi_{\text {puis }}\left(\mu_{\phi}\right), \mathcal{C}_{\text {ord }}\left(\mu_{\phi, t}, \nu_{\phi, t}\right)=\mathcal{C}_{\text {ord }}\left(\mu_{\phi}, \nu_{\phi}\right) ;
$$

and if we write $\mathcal{D}_{\mathcal{V}}(v, B, t):=\left(v_{t}, B_{t}, t\right)$, then $L\left(v_{t}\right)=L(v), h\left(B_{t}\right)=h(B)$.

\section{The Equi-Singular Deformation Theorem}

A real analytic map-germ $\rho:([0, \infty), 0) \rightarrow\left(\mathbb{C}^{2}, 0\right)$ is called an analytic arc $([17])$; we call the image set-germ, $\operatorname{Im}(\rho)$, a geo-arc. The complexification of $\rho$ is $\rho_{\mathbb{C}}(z):=\rho(z), z \in \mathbb{C}$.

Given $k \in \mathbb{Z}^{+}$, define $\rho^{(k)}(s):=\rho\left(s^{k}\right)$. Of course, $\operatorname{Im}\left(\rho^{(k)}\right)=\operatorname{Im}(\rho)$, the same geo-arc.

Let $f(x, y) \in \mathbb{C}\{x, y\}$ be given, mini-regular in $x$ of order $m$, i.e.,

$$
f(x, y)=H_{m}(x, y)+H_{m+1}(x, y)+\cdots, H_{m}(1,0) \neq 0 .
$$

Let $\phi(\xi):=f(\xi, y)$. In $\mathbb{C}_{*}$, define $\alpha_{*} \sim_{f} \beta_{*}$ iff either $\alpha_{*}=\beta_{*}$, or else

$$
\mathcal{C}_{\text {ord }}\left(\alpha_{*}, \beta_{*}\right)>\mathcal{C}_{\text {ord }}\left(\alpha_{*}, \zeta_{*}\right)=\mathcal{C}_{\text {ord }}\left(\beta_{*}, \zeta_{*}\right) \forall \zeta \in Z(\phi) \text {. }
$$

The equivalence class of $\alpha_{*}$ is denoted by $\alpha_{* / f}$. (If $\zeta \in Z(\phi), \zeta_{* / f}:=\left\{\zeta_{*}\right\}$.)

Call $\alpha_{\phi}(y)$, and any one of the conjugates $\alpha_{\phi, c o n j}^{(k)}(y)$, a canonical coordinate of $\alpha_{* / f}$.

We say $\alpha_{* / f}, \beta_{* / f}$ are bar equivalent: $\alpha_{* / f} \sim_{b a r} \beta_{* / f}$, if they have canonical coordinates $\alpha_{\phi}, \beta_{\phi}$ respectively, such that $\alpha_{\phi} \sim_{b a r} \beta_{\phi}$.

Example 3.1. Take $f(x, y):=x^{2}-2 y^{3}$. The curve-germs $x^{2}-y^{3}=0$ and $\left(x^{2}-y^{3}\right)^{2}-x y^{5}=0$ are $\sim_{f}$ equivalent, hence define a same point in $\mathbb{C}_{* / f}$, with canonical coordinates $\pm y^{3 / 2}$.

Definition 3.2. The valuation function on the quotient space $\mathbb{C}_{* / f}:=\mathbb{C}_{*} / \sim_{f}$ is

$$
\operatorname{val}_{* / f}: \mathbb{C}_{* / f} \rightarrow \mathcal{V}_{\mathbb{Z}}, \quad \alpha_{* / f} \mapsto\left[\prod_{k=1}^{N} \phi\left(\alpha_{\phi, \text { conj }}^{(k)}(y)\right)\right]_{i d}, \quad N:=m_{\text {puis }}\left(\alpha_{\phi}\right),
$$

where $\mathcal{V}_{\mathbb{Z}}:=\left\{0_{\mathcal{V}}\right\} \cup\left\{(u, h) \mid u \neq 0, h \in \mathbb{Z}^{+}\right\}$, a subspace of $\mathcal{V}$.

If $\phi^{\prime}(\gamma)=0, \gamma \in \mathbb{M}_{1}, \gamma_{* / f}$ is called a critical point of $v a l_{* / f}$, the multiplicity, $m_{\text {crit }}\left(\gamma_{* / f}\right)$, is the total number of $\mu \in \mathbb{M}_{1}$, counting multiplicities, such that $\phi^{\prime}(\mu)=0, \mu_{* / f}=\gamma_{* / f}$.

The subspace of critical points of $v a l_{* / f}$ is denoted by $C\left(v a l_{* / f}\right)$. 
In $\mathbb{C}_{* / f}$, define height by $h\left(\alpha_{* / f}\right):=h\left(\alpha_{\phi}\right)$, and contact order by

$$
\mathcal{C}_{\text {ord }}\left(\alpha_{* / f}, \beta_{* / f}\right):=\mathcal{C}_{\text {ord }}\left(\alpha_{*}, \beta_{*}\right) \text { if } \alpha_{* / f} \neq \beta_{* / f} ; \mathcal{C}_{\text {ord }}\left(\alpha_{* / f}, \alpha_{* / f}\right):=\infty \text {. }
$$

The Puiseux pairs are $\chi_{\text {puis }}\left(\alpha_{* / f}\right):=\chi_{\text {puis }}\left(\alpha_{\phi}\right)$.

Let $F(x, y, t):=F_{t}(x, y):=\sum_{i+j>m} c_{i j}(t) x^{i} y^{j} \in \mathbb{C}\{x, y, t\}$ be a given deformation of $f(x, y)$, i.e., $F_{0}(x, y)=f(x, y), F_{t}(0,0) \equiv 0$. In $\mathbb{C}_{*} \times I_{\mathbb{C}}$ define $\left(\alpha_{*}, t\right) \sim_{F}\left(\beta_{*}, t^{\prime}\right)$ iff $t=t^{\prime}$ and $\alpha_{* / F_{t}}=\beta_{* / F_{t}}$. The quotient space is $\mathbb{C}_{*} \times_{F} I_{\mathbb{C}}:=\mathbb{C}_{*} \times I_{\mathbb{C}} / \sim_{F}$.

The Equi-singular Deformation Theorem. Suppose the deformation $\Phi(\xi, t):=F(\xi, y, t)$ is almost Morse stable. Then there exists a t-level preserving homeomorphism

$$
H:\left(\mathbb{C}^{2} \times I_{\mathbb{C}}, 0 \times I_{\mathbb{C}}\right) \rightarrow\left(\mathbb{C}^{2} \times I_{\mathbb{C}}, 0 \times I_{\mathbb{C}}\right), \quad((x, y), t) \mapsto\left(H_{t}(x, y), t\right),
$$

which is real bi-analytic outside $\{0\} \times I_{\mathbb{C}}$. The following hold.

(1) $F\left(H_{t}(x, y), t\right)=f(x, y), t \in I_{\mathbb{C}}$, i.e., $F(x, y, t)$ is "trivialized" by $H$.

(2) There exists $c>0, c \leq\left\|H_{t}(x, y)\right\| /\|(x, y)\| \leq 1 / c, t \in I_{\mathbb{C}}$.

(3) If $\rho(s)$ is an analytic arc, then $H_{t}\left(\rho^{(k)}(s)\right)$ is real analytic in $(s, t)$ (called a "geo-arc wing") for some $k \in \mathbb{Z}^{+}$. In particular, $H_{t}$ is geo-arc analytic in the sense that it carries geo-arcs to geo-arcs.

(4) Take $\alpha_{* / f} \in \mathbb{C}_{* / f}$, and any $\rho$ such that $\operatorname{Im}\left(\rho_{\mathbb{C}}\right) \in \alpha_{* / f}$. The geo-arc $\operatorname{Im}\left(H_{t} \circ \rho\right)$ is contained in a unique curve-germ, say $\delta_{*} \in \mathbb{C}_{*}$. Then $\eta_{t}\left(\alpha_{* / f}\right):=\delta_{* / F_{t}}$ is independent of the choice of $\rho$. Hence $\eta_{t}: \mathbb{C}_{* / f} \rightarrow \mathbb{C}_{* / F_{t}}$ is well-defined,

$$
\eta_{*}: \mathbb{C}_{* / f} \times I_{\mathbb{C}} \rightarrow \mathbb{C}_{*} \times_{F} I_{\mathbb{C}}, \quad\left(\alpha_{* / f}, t\right) \mapsto\left(\eta_{t}\left(\alpha_{* / f}\right), t\right),
$$

is a homeomorphism, preserving height, contact order, and Puiseux pairs.

(5) If $\gamma_{* / f} \in C\left(v a l_{* / f}\right)$ then $\eta_{t}\left(\gamma_{* / f}\right) \in C\left(v a l_{* / F_{t}}\right), m_{\text {crit }}\left(\gamma_{* / f}\right)=m_{\text {crit }}\left(\eta_{t}\left(\gamma_{* / f}\right)\right)$.

Assume $\Phi(\xi, t)$ is Morse stable. Take $\gamma_{* / f}, \gamma_{* / f}^{\prime} \in C\left(v_{a l}\right), \gamma_{* / f} \sim_{b a r} \gamma_{* / f}^{\prime}$. Then

$$
v_{a l} l_{* / f}\left(\gamma_{* / f}\right)=\operatorname{val}_{* / f}\left(\gamma_{* / f}^{\prime}\right) \text { implies } \operatorname{val}_{* / F_{t}}\left(\eta_{t}\left(\gamma_{* / f}\right)\right)=\operatorname{val}_{* / F_{t}}\left(\eta_{t}\left(\gamma_{* / f}^{\prime}\right)\right) \text {, }
$$

where $\eta_{t}\left(\gamma_{* / f}\right) \sim_{b a r} \eta_{t}\left(\gamma_{* / f}^{\prime}\right)$.

Now let us consider $f(x, y), Z(\phi):=\left\{\zeta_{1}, \ldots, \zeta_{d}\right\}$ as in (2.5) $, m_{i}:=m\left(\zeta_{i}\right)$,

$$
f(x, y)=u(x, y) \cdot \prod_{i=1}^{d}\left(x-\zeta_{i}(y)\right)^{m_{i}}, \quad u(0,0) \neq 0 .
$$

Let $e_{i}:=\max _{j \neq i}\left\{O_{y}\left(\zeta_{i}-\zeta_{j}\right)\right\}$. Let $\hat{\zeta}_{i}(y)$ denote $\zeta_{i}(y)$ with all terms $y^{e}$ deleted, $e>e_{i}$.

Definition 3.3. The Puiseux root truncation of $f(x, y)$ is, by definition,

$$
\hat{f}_{\text {root }}(x, y):= \begin{cases}\prod_{i=1}^{d}\left(x-\hat{\zeta}_{i}(y)\right)^{m_{i}} & \text { if } d>1, \\ \left(x-\zeta_{1}\right)^{m_{1}} & \text { if } d=1 .\end{cases}
$$

Let $R_{i}(y):=\zeta_{i}(y)-\hat{\zeta}_{i}(y)$ (the remainder). Take $u(x, y, t)$ (a deformation of unit),

$$
u(0,0, t) \neq 0, \quad u(x, y, 0)=u(x, y), \quad u(x, y, 1)=1 .
$$


The Puiseux root deformation of $f(x, y)$ is, by definition,

$$
F_{\text {root }}(x, y, t):=u(x, y, t) \cdot \prod_{i=1}^{d}\left[x-\zeta_{i}(y)+t R_{i}(y)\right]^{m_{i}} \in \mathbb{C}\{x, y, t\} .
$$

Note that $\hat{f}_{\text {root }}(x, y) \in \mathbb{C}\{x, y\}$, since it is invariant under the conjugations; if $d>1$ then $\hat{f}_{\text {root }}(x, y)$ is a polynomial. The following theorem is proved at the end of $₫ 9$.

The Truncation Theorem. The Puiseux root deformation $F_{\text {root }}(x, y, t)$ is Morse stable. In particular, $f(x, y)$ and $\hat{f}_{\text {root }}(x, y)$ are geo-arc analytically equivalent.

Example 3.4. For a weighted expansion $f(x, y):=W_{d}(x, y)+\cdots$, if $W_{d}$ is non-degenerate then $\hat{f}_{\text {root }}(x, y)=W_{d}(x, y), f(x, y)$ is geo-arc analytically equivalent to its initial form.

Next, $g(x, y):=\left(x^{2}-y^{4}\right)^{2}-y^{10}+\cdots$ has Puiseux roots $x= \pm y^{2} \pm \frac{1}{2} y^{3}+\cdots$,

$$
\hat{g}_{\text {root }}(x, y)=\left(x^{2}-y^{4}\right)^{2}-\frac{1}{4} y^{6}\left[\left(x-y^{2}\right)^{2}+\left(x+y^{2}\right)^{2}\right]+\frac{1}{16} y^{12} .
$$

Note that $\hat{g}_{\text {root }}(x, y)$ is not obtained by deleting certain terms of $g(x, y)$.

Remark 3.5. We call $\alpha_{* / f}$ an " $f$-blurred" infinitesimal: Points of $\mathbb{C}_{*}$ equivalent under $\sim_{f}$ are no longer distinguishable - "f-blurred". The notion of blurring plays a vital role in this paper. For example, the Pham deformations

$$
P_{\text {even }}(x, y, t)=x^{3}-y^{4}+3 t x y^{2 d}, \quad P_{\text {odd }}(x, y, t)=x^{3}-y^{4}+3 t x y^{2 d+1},
$$

where $d \geq 2$, are regarded by some experts as substantially different, since the polars are very different when $t \neq 0$. To us, however, there is only one critical point - a "blurred polar" - in either case, of multiplicity 2. (Compare Attention8.2). The above theorem applies.

Remark 3.6. The above (3.3) says that the family $\left\{v a l_{* / F_{t}}\right\}$ on the spaces $\left\{\mathbb{C}_{* / F_{t}}\right\}$ is Morse stable, the deformation of the critical points being given by $\eta_{t}$. Hence, as in the classical case, we can construct a trivialization of the family, like $\left(D_{t}, d_{t}\right)$, in $\S[10$.

However, we are not saying that this trivialization coincides with $\eta_{*}$. We believe it would be too good (too strong) for this to be true.

\section{Proof Of Cauchy's Theorem}

Let $\delta \in \mathbb{D}$ be given, and fixed. As $\phi$ is Puiseux-Lojasiewicz bounded, we can write

$$
y^{L} \phi(\alpha+z \delta)=\sum_{i=0}^{\infty} c_{\delta, i}(z) y^{n_{i} / K}, \quad 0<K \leq n_{0}<\cdots
$$

where $L, K$ are constants, $|z|$ sufficiently small.

Take an increment $\Delta z$ and compute the derivative. We find

$$
y^{L} \phi^{\prime}(\alpha+z \delta)=\frac{1}{\delta} \sum c_{\delta, i}^{\prime}(z) y^{n_{i} / K}
$$


In particular, $c_{\delta, i}^{\prime}(z)$ exists, $c_{\delta, i}(z)$ is holomorphic,

$$
c_{\delta, i}(0)=\frac{1}{2 \pi \sqrt{-1}} \oint_{z \in C} \frac{c_{\delta, i}(z)}{z} d z, \quad \phi(\alpha)=\frac{1}{2 \pi \sqrt{-1}} \oint_{z \in C} \frac{\phi(\mu)}{\mu-\alpha} d \mu,
$$

where $\mu:=\alpha+z \delta$. Then, as in Complex Analysis, (2.1) follows.

Next we show (2.2). Take $\delta=1$ in (4.1), and then set $z=0$. We have

$$
y^{L} \phi^{\prime}(\alpha+z)=\sum c_{1, i}^{\prime}(z) y^{n_{i} / K}, \quad c_{1, i}^{\prime}(0)=\frac{1}{\delta} c_{\delta, i}^{\prime}(0)
$$

Applying the same argument to higher derivatives, we have

$$
c_{1, i}^{(k)}(0)=\delta^{-k} c_{\delta, i}^{(k)}(0), \quad k \geq 1
$$

Hence,

$$
y^{L} \phi(\alpha+z \delta)=\sum_{i} \sum_{k} \frac{1}{k !} c_{\delta, i}^{(k)}(0) z^{k} y^{n_{i} / K}=\sum_{k} \frac{1}{k !} \phi^{(k)}(\alpha) \cdot(z \delta)^{k} .
$$

Take $\delta=y^{h}, h \in \mathbb{Q}^{+}$. If $m_{\text {puis }}\left(\phi^{(k)}(\alpha)\right)$ were unbounded, then $m_{\text {puis }}\left(\phi\left(\alpha+c y^{h}\right)\right)=\infty$ for generic $c$, a contradiction. This completes the proof.

Corollary 4.1. Suppose $\phi: U \rightarrow \mathbb{D}$ is $\mathbb{F}$-analytic, $0 \in U, \phi(0)=0$. Then there exist a holomorphic germ $f(x, y)$ and $N \in \mathbb{Z}^{+}$such that $\phi(\xi)=f\left(\xi, y^{1 / N}\right), \xi \in \mathbb{M}$.

Proof. Take $\alpha=0, S:=\sup _{k}\left\{m_{\text {puis }}\left(\phi^{(k)}(0)\right)\right\}, N:=S$ !. All $\phi^{(k)}(0)\left(y^{N}\right)$ in (2.2) are integral power series of $y, f(x, y):=\sum(1 / k !) \phi^{(k)}(0)\left(y^{N}\right) x^{k}$ is holomorphic $0, f(0,0)=0$.

\section{Newton Polygon At An Infinitesimal}

Given $\phi$ and $\alpha$. For a term $\alpha_{k} \xi^{k} \neq 0$ in (2.3), plot a "Newton dot" at $(k, q)$ in the $(u, v)$ plane, $q:=O_{y}\left(\alpha_{k}\right)$. Consider the convex hull generated by $\left\{\left(a_{i}+u, b_{i}+v\right) \mid u, v \geq 0\right\},\left(a_{i}, b_{i}\right)$ the Newton dots. The boundary $\mathcal{N P}(\phi, \alpha)$ is the Newton Polygon of $\phi$ at $\alpha$. (See [14].)

In the case $\phi(\xi, y):=f(\xi, y), f(x, y) \in \mathbb{C}\{x, y\}$, we clearly have $\mathcal{N} \mathcal{P}(\phi, \alpha)=\mathcal{N} \mathcal{P}\left(\phi, \alpha_{\text {conj }}^{(k)}\right)$. Hence $\mathcal{N} \mathcal{P}\left(\phi, \alpha_{*}\right):=\mathcal{N} \mathcal{P}\left(\phi, \alpha_{\text {conj }}^{(k)}\right)$ is well-defined. This is the Newton Polygon of $\phi$ at $\alpha_{*}$.

Consider $\mathcal{N} \mathcal{P}(\phi, \alpha)$. The edges and angles are $E_{i}:=E_{i}(\alpha), \theta_{i}:=\theta_{E_{i}}$, respectively, $\theta_{i-1}<\theta_{i}$, $0 \leq i \leq l$. The first edge $E_{0}$ is horizontal, the last edge $E_{l}$ is vertical. Denote the right vertex of $E_{i}$ by $V_{i}:=\left(m_{i}, q_{i}\right)$, and the straight line prolonging $E_{i}$ by $\mathcal{L}\left(E_{i}\right)$.

The vertical edge $E_{l}$ is not important. We call $E_{t o p}:=E_{l-1}$ the top Newton edge. The left vertex of $E_{l-1}$ is $\left(m_{l}, q_{l}\right)$, which is the last, and the highest, vertex of $\mathcal{N} \mathcal{P}(\phi, \alpha)$.

Take $E_{i}, i \leq l-1$. A $\operatorname{dot}(k, q) \in E_{i}$ represents a term $c_{k} y^{q} \xi^{k}$ of (2.3). Let

$$
W_{E_{i}}(\xi, y):=\sum c_{k} y^{q} \xi^{k}, \quad P_{E_{i}}(z):=\sum c_{k} z^{k} \in \mathbb{C}[z],
$$

sum taken over $(k, q) \in E_{i}$. We call $P_{E_{i}}(z)$ the associated polynomial of $E_{i}$.

The height, or co-slope, of $E_{i}$ is

$$
h\left(E_{i}\right):=\tan \theta_{i}, \quad 0 \leq i \leq l-1 ; \quad h\left(E_{l}\right):=\tan \theta_{l}=\infty .
$$


The Lojasiewicz exponent on $E_{i}$ is

$$
L\left(E_{i}\right):=q_{i}+m_{i} \tan \theta_{i}, \quad 0 \leq i \leq l-1 ; \quad L\left(E_{l}\right):=\infty .
$$

Example 5.1. (Fig.1,2) Take $\alpha=0$. For $\phi_{1}(\xi):=\xi^{3}+2 y \xi^{2}+y^{4}, \tan \theta_{1}=1, \tan \theta_{\text {top }}=3 / 2$, $P_{E_{1}}(z)=z^{3}+2 z^{2}, P_{t o p}(z)=2 z^{2}+1$. For $\phi_{2}(\xi):=\xi^{3}+2 y \xi^{2}, P_{\text {top }}(z)=z^{3}+2 z^{2}, E_{2}=E_{l}$.

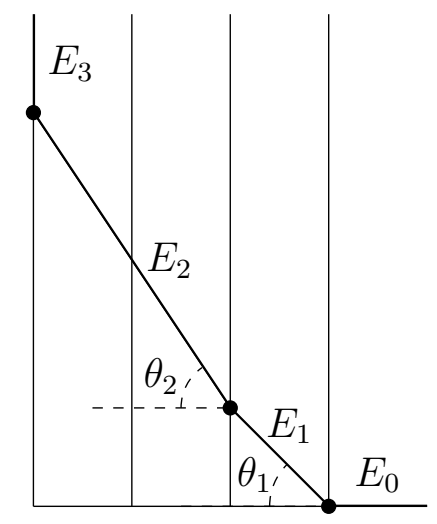

FiguRE 1. $\mathcal{N} \mathcal{P}\left(\phi_{1}, 0\right)$

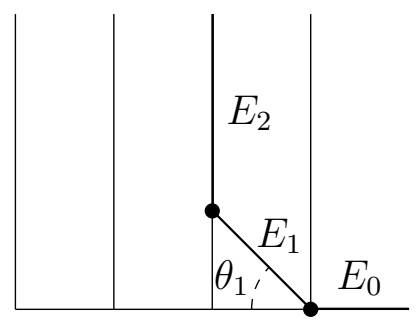

Figure 2. $\mathcal{N} \mathcal{P}\left(\phi_{2}, 0\right)$

Notation 5.2. Suppose $\mathcal{N} \mathcal{P}(\phi, \alpha), \mathcal{N} \mathcal{P}(\phi, \beta)$ have a common edge $E_{k}(\alpha)=E_{k}(\beta)$.

We write $E_{k}(\alpha) \equiv E_{k}(\beta)$ if they have the same Newton dots, each represents a same monomial term of $\phi$. We write $\mathcal{N} \mathcal{P}(\phi, \alpha) \equiv \mathcal{N} \mathcal{P}(\phi, \beta)$ if this is true for every edge.

Observe that if $\alpha \notin Z(\phi)$, then $m_{l}=0$, and

$$
h\left(E_{l-1}\right)=\max \left\{O\left(\alpha-\zeta_{i}\right)\right\}, \quad \zeta_{i} \in Z(\phi) .
$$

If $\alpha \sim_{\phi} \beta$, then $O(\alpha-\beta)>h\left(E_{l-1}\right)$, and hence $\mathcal{N} \mathcal{P}(\phi, \alpha) \equiv \mathcal{N} \mathcal{P}(\phi, \beta)$. Thus, $P_{E_{i}\left(\alpha_{\phi}\right)}(z)$, $\mathcal{N} \mathcal{P}\left(\phi, \alpha_{\phi}\right), E_{i}\left(\alpha_{\phi}\right)$, etc., are all well-defined (independent of the choice of $\left.\alpha \in \alpha_{\phi}\right)$.

(Similarly, $\mathcal{N} \mathcal{P}\left(f, \alpha_{* / f}\right):=\mathcal{N} \mathcal{P}\left(\phi, \alpha_{\phi}\right)$ is also well-defined.)

Theorem 5.3. Take an edge $E_{i}$ of $\mathcal{N} \mathcal{P}\left(\phi, \alpha_{\phi}\right), 1 \leq i \leq l-1$. Take a critical point $c$ of the associated polynomial $P_{E_{i}}(z)$, with multiplicity $m_{c r i t}(c)$. Then there are exactly $m_{c r i t}(c)$ critical points of $\phi$ in $\mathbb{M}_{1}$, counting multiplicities, of the form

$$
\mu(y)=\hat{\alpha}_{\phi}(y)+\left[c y^{\tan \theta_{i}}+\cdots\right]
$$

where $\hat{\alpha}_{\phi}$ is $\alpha_{\phi}(y)$ with all terms $y^{e}$ (if any) deleted, $e>\tan \theta_{i}$.

Take $\mu_{\phi} \in C\left(\right.$ val $\left._{\phi}\right), \mu_{\phi} \neq \alpha_{\phi}$. There exist a unique $E_{i}$ and a unique critical point $c \neq 0$ of $P_{E_{i}}(z)$ such that $\mu(y)$ has the form (5.2).

According to Convention 1.1, we can also write (5.2) as

$$
\mu(y)=\alpha_{\phi}(y)+\left[c y^{\tan \theta_{i}}+\cdots\right] .
$$

Theorem 5.3 is known ([13], see also [14]). We use it several times in this paper. 
The Fundamental Lemma. Suppose $\Phi$ is almost Morse stable. Then

$$
\mathcal{N} \mathcal{P}\left(\phi, \gamma_{\phi}\right)=\mathcal{N} \mathcal{P}\left(\phi_{t}, \tau_{t}\left(\gamma_{\phi}\right)\right), \quad \chi_{\text {puis }}\left(\tau_{t}\left(\gamma_{\phi}\right)\right)=\chi_{\text {puis }}\left(\gamma_{\phi}\right)
$$

where $\gamma_{\phi} \in C\left(\right.$ val $\left._{\phi}\right)$ with deformation $\tau_{t}\left(\gamma_{\phi}\right) \in C\left(\right.$ val $\left._{\phi_{t}}\right)$, as in (2.8).

The canonical coordinate $\tau_{t}\left(\gamma_{\phi}\right)(y)$ of $\tau_{t}\left(\gamma_{\phi}\right)$ is an $\mathbb{F}$-analytic function of $t \in I_{\mathbb{F}}$. For each $E_{i}, i \leq l-1$, the family $\left\{P_{E_{i}\left(\tau_{t}\left(\gamma_{\phi}\right)\right)}(z)\right\}$ is almost Morse stable, as defined below.

Moreover, if $\Phi$ is Morse stable, then so is the family $\left\{P_{E_{i}\left(\tau_{t}\left(\gamma_{\phi}\right)\right)}(z)\right\}$.

Definition 5.4. Given a polynomial $p(z)$ and a deformation

$$
p_{t}(x):=a_{0}(t) x^{n}+\cdots+a_{n}(t) \in \mathbb{K}\{t\}[x], \quad p_{0}(z)=p(z), \quad a(t) \neq 0,
$$

where $\mathbb{K}:=\mathbb{R}, \mathbb{C}$, or $\mathbb{F}, t \in I_{\mathbb{K}}$.

A critical point $c_{0} \in \mathbb{K}$ of $p_{0}(x)$ is stable if it admits a continuous deformation $c_{t} \in \mathbb{K}$ such that $p_{t}^{\prime}\left(c_{t}\right)=0$ and $m_{\text {crit }}\left(c_{t}\right)=m_{\text {crit }}\left(c_{0}\right)$. (The deformation is then necessarily unique.)

Consider the following conditions (where (3) is for Algebraic Geometry):

(1) Every critical point of $p_{0}(x)$ is stable.

(2) If $c_{0}, c_{0}^{\prime}$ are critical points of $p_{0}(x)$ and $p_{0}\left(c_{0}\right)=p_{0}\left(c_{0}^{\prime}\right)$, then $p_{t}\left(c_{t}\right)=p_{t}\left(c_{t}^{\prime}\right)$.

(3) If $p_{0}\left(c_{0}\right)=p_{0}^{\prime}\left(c_{0}\right)=0$, i.e., $c_{0}$ is a multiple root of $p_{0}(z)$, then $p_{t}\left(c_{t}\right)=0$.

We say $\left\{p_{t}\right\}$ is almost Morse stable if (1), (3) hold, and Morse stable if (2) also holds.

Example 5.5. Take $\mathbb{K}=\mathbb{R}$. For $p_{t}(x)=x^{2}\left(x^{2}+t^{2}\right) \in \mathbb{R}[x], 0$ is a critical point of $p_{0}$ which splits into three critical points in $\mathbb{C}$, one remains in $\mathbb{R}$. Thus 0 admits a unique continuous deformation $c_{t} \equiv 0$ in $\mathbb{R}$. But $m_{\text {crit }}\left(c_{t}\right)$ is not constant, 0 is unstable.

Proof. We use the "edging forward argument" to prove (5.3). The Tschirnhausen transformation is applied recursively along the edges of $\mathcal{N} \mathcal{P}\left(\phi_{t}, \gamma_{\phi}\right)$ ("edging forward") in order to "clear" all dots of $\phi_{t}$ lying below $\mathcal{N} \mathcal{P}\left(\phi, \gamma_{\phi}\right)$. If $m_{l}>0$, all dots to the left of the vertical edge $E_{l}$ are also cleared. The details are as follows.

Consider $\mathcal{N} \mathcal{P}\left(\phi, \gamma_{\phi}\right)$. The edges are denoted by $E_{i}$. The right vertex of $E_{i}$ is $\left(m_{i}, q_{i}\right)$.

Let us first compare $\mathcal{N} \mathcal{P}\left(\phi, \gamma_{\phi}\right)$ with $\mathcal{N} \mathcal{P}\left(\phi_{t}, \gamma_{\phi}\right)$. Write

$$
\phi_{t}\left(\gamma_{\phi}+\xi\right)=\phi\left(\gamma_{\phi}+\xi\right)+P_{t}\left(\gamma_{\phi}+\xi\right), \quad P_{0}\left(\gamma_{\phi}+\xi\right) \equiv 0 .
$$

The (non-zero) terms of $P_{t}$ are represented by dots. Some may lie below $\mathcal{N} \mathcal{P}\left(\phi, \gamma_{\phi}\right)$.

Suppose we already know that $P_{t}\left(\gamma_{\phi}+\xi\right)$ has no dot below the lines $\mathcal{L}\left(E_{j}\right), 0 \leq j \leq k-1$. We can then clear the dots under the line $\mathcal{L}\left(E_{k}\right)$ as follows.

The left vertex of $E_{k-1}$ represents a term $a y^{q_{k}} \xi^{m_{k}}$ of $\phi\left(\gamma_{\phi}+\xi\right)$, which, with a Tschirnhausen transformation, can "swallow" all dots of $P_{t}$ of the form $\left(m_{k}-1, q\right), q \in \mathbb{Q}^{+}$.

This means the following. There exists $\beta_{t}(y) \in \mathbb{M}_{1}, \mathbb{F}$-analytic in $t$, such that

(1) $O_{y}\left(\beta_{t}(y)\right) \geq \tan \theta_{k-1}, \beta_{0}(y)=0$;

(2) The coefficient of $\xi^{m_{k}-1}$ in the Taylor expansion of $\phi\left(\gamma_{\phi}+\beta_{t}+\xi\right)$ is independent of $t$.

Indeed, $\gamma_{\phi}+\xi \mapsto \gamma_{\phi}+\xi+\beta_{t}$ is the unique translation (Tschirnhausen transformation) which has the above two properties. (Attention: No dot of $\phi\left(\gamma_{\phi}+\xi\right)$ has been swallowed in the process. In this way, we have $\beta_{0}=0$. This property is important.) 
Now, consider $\mathcal{N} \mathcal{P}\left(\phi_{t}, \beta_{t}+\gamma_{\phi}\right)$. Because of $(1), E_{j}\left(\gamma_{\phi}\right) \equiv E_{j}\left(\gamma_{\phi}+\beta_{t}\right), j \leq k-2$, (we use Notation 5.2, ) and $P_{t}$ still has no dot below the line $\mathcal{L}\left(E_{j}\right), j \leq k-1$.

Let $E_{k-1}^{\prime}, E_{k}^{\prime}, \ldots$, denote the remaining edges of $\mathcal{N} \mathcal{P}\left(\phi_{t}, \beta_{t}+\gamma_{\phi}\right)$. By $(1), \theta_{E_{k-1}}=\theta_{E_{k-1}^{\prime}}$.

Next we show $E_{k-1}=E_{k-1}^{\prime}$. Let $V_{j}^{\prime}:=\left(m_{j}^{\prime}, q_{j}^{\prime}\right)$ denote the right vertex of $E_{j}^{\prime}$.

Suppose $E_{k-1}^{\prime} \neq E_{k-1}$. Then $m_{k}^{\prime} \leq m_{k}-2$. We shall derive a contradiction.

For generic $t \in \mathbb{C}, 0$ is a root of $P_{E_{k-1}^{\prime}}(z)$ of multiplicity $m_{k}^{\prime}\left(m_{k}^{\prime} \geq 0\right)$, but when $t=0$, the multiplicity is $m_{k}$. Hence, by an elementary argument, there exists $a(t)$ such that

$$
\frac{d}{d z} P_{E_{k-1}^{\prime}}(a(t))=0, \quad P_{E_{k-1}^{\prime}}(a(t)) \neq 0, \quad \lim _{t \rightarrow 0} a(t)=0 .
$$

Thus, by Theorem 5.3, $\phi_{t}$ has a critical point of the form

$$
\Gamma_{t}(y):=\left[\beta_{t}(y)+\gamma_{\phi}(y)\right]+\left[a(t) y^{e}+\cdots\right], \quad e:=\tan \theta_{E_{k-1}} .
$$

Hence $h\left(\Gamma_{t, \phi_{t}}\right)=\tan \theta_{E_{k-1}}, L_{\phi_{t}}\left(\Gamma_{t, \phi_{t}}\right)=q_{k}+m_{k} \tan \theta_{E_{k-1}}$, both are independent of $t$.

Take $\mu_{\phi} \in C\left(v a l_{\phi}\right), \mu_{\phi} \neq \gamma_{\phi}$. Then take $E_{i}, c \neq 0$, for $\mu_{\phi}$ as in (5.2) with $\gamma_{\phi}$ replacing $\alpha_{\phi}$. We say $\mu_{\phi}$ is of the lower kind if $i \leq k-1$, and of the higher kind if $i \geq k$.

If $\mu_{\phi}=\gamma_{\phi}$, we say $\mu_{\phi}$ is of the higher kind.

Take $\varepsilon$, sufficiently small. The $\varepsilon$-neighborhood $\mathcal{N}_{\varepsilon}\left(\gamma_{\phi}\right)$ of $\gamma_{\phi}$ clearly does not contain any $\mu_{\phi}$ of the lower kind. On the other hand, if $|t|$ is sufficiently small, then, by continuity, $\Gamma_{t, \phi_{t}} \in \mathcal{N}_{\varepsilon}\left(\gamma_{\phi}\right)$. Hence $\Gamma_{t, \phi_{t}} \neq \tau_{t}\left(\mu_{\phi}\right)$ for any $\mu_{\phi}$ of the lower kind.

If $\mu_{\phi}$ is of the higher kind, then $L\left(\mu_{\phi}\right)=L\left(\tau_{t}\left(\mu_{\phi}\right)\right)>L\left(\Gamma_{t, \phi_{t}}\right)$. Hence $\Gamma_{t, \phi_{t}} \neq \tau_{t}\left(\mu_{\phi}\right)$ for any $\mu_{\phi}$ of the higher kind either. Thus we must have $E_{k-1}^{\prime}=E_{k-1}$.

Next we show $\theta_{E_{k}^{\prime}}=\theta_{E_{k}}$. Suppose $\theta_{E_{k}^{\prime}}<\theta_{E_{k}}$. Then $m_{k+1}^{\prime} \leq m_{k}-2$, and there would exist $a(t)$ as above. Using the same argument we again arrive at a contradiction.

Hence the Tschirnhausen transformation $\xi \mapsto \xi+\beta_{t}$ clears all dots of $P_{t}$ below $\mathcal{L}\left(E_{k}\right)$.

A recursive application of the Tschirnhausen transformations, beginning with $k=1$, clears all dots of $P_{t}$ below $\mathcal{N} \mathcal{P}\left(\phi, \gamma_{\phi}\right)$.

Let $\xi \mapsto \xi+B_{t}$ denote their composition. We then compare the polygons:

$$
\mathcal{N} \mathcal{P}^{(0)}:=\mathcal{N} \mathcal{P}\left(\phi, \gamma_{\phi}\right), \mathcal{N} \mathcal{P}^{(1)}:=\mathcal{N} \mathcal{P}\left(\phi_{t}, \gamma_{\phi}+B_{t}\right), \mathcal{N P}^{(2)}:=\mathcal{N} \mathcal{P}\left(\phi_{t}, \tau_{t}\left(\gamma_{\phi}\right)\right)
$$

We have just proved $\mathcal{N} \mathcal{P}^{(0)}=\mathcal{N} \mathcal{P}^{(1)}$. Next we show $\mathcal{N} \mathcal{P}^{(1)}=\mathcal{N} \mathcal{P}^{(2)}$.

We can assume $B_{t}=0$. This can be achieved by the substitution $\xi \rightarrow \xi+B_{t}(y)$.

Let us write $P_{\text {top }}^{(1)}(z):=P_{\text {top }}^{(0)}(z)+Q_{t}(z), Q_{0}(z) \equiv 0$, where 0 is a critical point of $P_{\text {top }}^{(0)}(z)$.

As $t$ varies away from 0 , this critical point cannot split into two or more critical points of $P_{\text {top }}^{(1)}(z)$. For if it did, the homeomorphism $\tau_{t}$ cannot exist.

Hence 0 admits a unique continuous deformation $c_{t}, c_{0}=0$, which is a critical point of $P_{\text {top }}^{(1)}(z), m_{\text {crit }}\left(c_{t}\right)=m_{\text {puis }}\left(c_{0}\right)$. It follows that $c_{t}$ is a simple root of the equation

$$
\frac{d^{m}}{d z^{m}} P_{\text {top }}^{(1)}(z)=0, \quad m:=m_{\text {crit }}\left(c_{0}\right),
$$

and

$$
\tau_{t}\left(\gamma_{\phi}\right)(y)=\gamma_{\phi}(y)+c_{t} y^{e}, \quad e:=\tan \theta_{t o p}^{(1)} .
$$


It follows that $c_{t}, \tau_{t}\left(\gamma_{\phi}\right)(y)$ are $\mathbb{F}$-analytic. (The Implicit Function Theorem holds in $\mathbb{F}$.) Let the Taylor expansion of $\phi_{t}$ at $\gamma_{\phi}$ be $\sum_{k, q} c_{q k}(t) y^{q} \xi^{k}$. Then that at $\tau_{t}\left(\gamma_{\phi}\right)$ is

$$
\sum c_{k q}(t) y^{q}\left[\xi+c_{t} y^{e}\right]^{k}=\sum c_{k q}(t)\left[y^{q} \xi^{k}+\cdots\right], \quad c_{0}=0
$$

Consider $E_{i}^{(1)}, i \leq l-2$. Since $e>\tan \theta_{i}$, the terms in " $+\cdots$ " are represented by dots lying strictly above all $E_{i}^{(1)}$. Hence $E_{i}^{(1)} \equiv E_{i}^{(2)}, i \leq l-2$. (See Notation 5.2.)

Now we show $E_{\text {top }}^{(1)}=E_{\text {top }}^{(2)}$. (But not $E_{\text {top }}^{(1)} \equiv E_{\text {top }}^{(2)}$.)

First suppose $P_{t o p}^{(0)}(0) \neq 0$. The left vertex of $E_{t o p}^{(0)}=E_{t o p}^{(1)}$ lies on the vertical coordinate axis. Hence $P_{\text {top }}^{(1)}(0) \neq 0, P_{\text {top }}^{(1)}\left(c_{t}\right) \neq 0(|t|$ small $)$. But $P_{\text {top }}^{(2)}(0)=P_{\text {top }}^{(1)}\left(c_{t}\right)$, hence $E_{\text {top }}^{(1)}=E_{\text {top }}^{(2)}$.

Suppose $P_{\text {top }}^{(0)}(0)=0$, i.e., 0 is a multiple root. As $\mathcal{N} \mathcal{P}^{(0)}=\mathcal{N} \mathcal{P}^{(1)}$, we must have

$$
c_{t} \equiv 0, \quad m_{\text {crit }}\left(c_{t}\right)=m_{l}=m_{\text {puis }}\left(\tau_{t}\left(\gamma_{\phi}\right)\right) .
$$

Hence $E_{\text {top }}^{(1)}=E_{\text {top }}^{(2)}$, and $\mathcal{N} \mathcal{P}^{(1)}=\mathcal{N} \mathcal{P}^{(2)}$.

It is easy to see that $\chi_{\text {puis }}\left(\alpha_{\phi}\right)$ can be expressed in terms of the co-slopes of the edges of $\mathcal{N P}\left(\phi, \alpha_{\phi}\right)$. It follows that $\chi_{\text {puis }}\left(\tau_{t}\left(\gamma_{\phi}\right)\right)=\chi_{\text {puis }}\left(\gamma_{\phi}\right)$. This completes the proof of (5.3).

Now assume $\Phi$ is Morse stable. We show $\left\{P_{E_{i}\left(\tau_{t}\left(\gamma_{\phi}\right)\right)}(z)\right\}$ is Morse stable.

Take a critical point $c$ of $P_{E_{i}\left(\gamma_{\phi}\right)}(z)$. Take $\mu$ as in (5.2). Consider $\mu_{\phi}, \mathcal{N} \mathcal{P}\left(\phi, \mu_{\phi}\right)$, etc..

Note that $P_{E_{i}\left(\gamma_{\phi}\right)}(z+c)=P_{E_{i}\left(\mu_{\phi}\right)}(z)$ (differ merely by a translation).

First, if $P_{E_{i}\left(\gamma_{\phi}\right)}(c) \neq 0$, then $P_{E_{i}\left(\mu_{\phi}\right)}(0) \neq 0$. Hence $E_{i}\left(\mu_{\phi}\right)$ has its left vertex on the vertical axis, 0 being a critical point of $P_{E_{i}\left(\mu_{\phi}\right)}(z)$. Then, as in the argument for $\gamma_{\phi}, 0$ admits a unique continuous deformation which is a critical point of $P_{E_{i}\left(\tau_{t}\left(\mu_{\phi}\right)\right.}(z)$ with constant multiplicity. This says that $c$ is a stable critical point of $P_{E_{i}\left(\tau_{t}\left(\gamma_{\phi}\right)\right)}(z)$.

Suppose $P_{E_{i}\left(\gamma_{\phi}\right)}(c)=0$, say of multiplicity $k$. Then 0 is a root of $P_{E_{i}\left(\mu_{\phi}\right)}(z)$, also of multiplicity $k$. Since $\mathcal{N} \mathcal{P}\left(\phi, \mu_{\phi}\right)=\mathcal{N} \mathcal{P}\left(\phi_{t}, \tau_{t}\left(\mu_{\phi}\right)\right)$, 0 is obviously stable. Hence so is $c$.

Now we show (2) in Definition 5.4. Let us write $p_{t}(z):=P_{E_{i}\left(\tau_{t}\left(\gamma_{\phi}\right)\right)}(z)$. Let $c \neq c^{\prime}$ be critical points of $p_{0}(z)$. Take $\mu, \mu^{\prime}$ for $E_{i}, c$ and $c^{\prime}$ respectively, $\mu_{\phi}, \mu_{\phi}^{\prime} \in C\left(v a l_{\phi}\right)$.

First, suppose $p_{0}(c)=p_{0}\left(c^{\prime}\right) \neq 0$. In this case,

$$
h\left(\mu_{\phi}\right)=h\left(\mu_{\phi}^{\prime}\right)=O\left(\mu_{\phi}-\mu_{\phi}^{\prime}\right)=\tan \theta_{E_{i}} .
$$

Hence $B\left(\mu_{\phi}\right)=B\left(\mu_{\phi}^{\prime}\right), v a l_{\phi}\left(\mu_{\phi}\right)=v_{a l}\left(\mu_{\phi}^{\prime}\right)$. Then, by (2.9), $\operatorname{val}_{\phi_{t}}\left(\tau_{t}\left(\mu_{\phi}\right)\right)=\operatorname{val}_{\phi_{t}}\left(\tau_{t}\left(\mu_{\phi}^{\prime}\right)\right)$. That is, $\left(p_{t}\left(c_{t}\right), \tan \theta_{E_{i}}\right)=\left(p_{t}\left(c_{t}^{\prime}\right), \tan \theta_{E_{i}}\right) \in \mathcal{V}$. In particular, $p_{t}\left(c_{t}\right)=p_{t}\left(c_{t}^{\prime}\right)$.

Suppose $p_{0}(c)=p_{0}\left(c^{\prime}\right)=0$. Then $c, c^{\prime}$ are multiple roots of $p_{0}$, and, as shown before, their deformations remain multiple roots of $p_{t}, p_{t}\left(c_{t}\right)=p_{t}\left(c_{t}^{\prime}\right)=0$.

By the same argument, if $\Phi$ is almost Morse stable then so are the families $\left\{P_{E_{i}\left(\tau_{t}\left(\gamma_{\phi}\right)\right)}\right\}$.

Corollary 5.6. Let $\left.Z(\Phi):=\left\{\left(\zeta_{t}, t\right)\right) \mid \zeta_{t} \in Z\left(\phi_{t}\right)\right\}$. There exists a bijection

$$
\mathcal{D}: Z(\phi) \times I_{\mathbb{F}} \rightarrow Z(\Phi), \quad(\zeta, t) \mapsto\left(\zeta_{t}, t\right), \quad \zeta_{0}=\zeta
$$

where $t \mapsto \zeta_{t}$ is $\mathbb{F}$-analytic; the Newton Polygon $\mathcal{N} \mathcal{P}\left(\phi_{t}, \zeta_{t}\right)$ is independent of $t$.

Take $\zeta, \zeta^{\prime} \in Z(\phi), \gamma_{\phi} \in C\left(v_{a l}\right)$. Then $O_{y}\left(\zeta_{t}-\zeta_{t}^{\prime}\right), O_{y}\left(\zeta_{t}-\tau_{t}\left(\gamma_{\phi}\right)\right)$ are independent of $t$. 
Proof. If $\zeta$ is a multiple root, $\zeta=\gamma_{\phi}$, then $\zeta_{t}=\tau_{t}\left(\gamma_{\phi}\right)$ is the deformation.

Otherwise, we choose $\gamma_{\phi} \in C\left(v_{a l}\right)$ such that

$$
O\left(\zeta-\gamma_{\phi}\right) \geq O\left(\zeta-\mu_{\phi}\right), \quad \forall \mu_{\phi} \in C\left(v a l_{\phi}\right) .
$$

We then have $h\left(\gamma_{\phi}\right)=O\left(\zeta-\gamma_{\phi}\right)$, and

$$
\zeta(y)=\gamma_{\phi}(y)+\left[b y^{h\left(\gamma_{\phi}\right)}+\cdots\right], \quad P_{E\left(\gamma_{\phi}\right)}(b)=0 \neq P_{E\left(\gamma_{\phi}\right)}^{\prime}(b) .
$$

(If $P_{E\left(\gamma_{\phi}\right)}^{\prime}(b)=0$, then there would exist $\mu_{\phi}$ which fails (5.5).) Using the Implicit Function Theorem we can find $\zeta_{t}$, which is $\mathbb{F}$-analytic, and $O\left(\zeta_{t}-\tau_{t}\left(\gamma_{\phi}\right)\right)$ is constant.

Given $\zeta^{\prime}$. Take $\gamma_{\phi}^{\prime}$ as in (5.5). Then $O\left(\tau_{t}\left(\gamma_{\phi}\right)-\tau_{t}\left(\gamma_{\phi}^{\prime}\right)\right)$ is constant, so is $O\left(\zeta_{t}-\zeta_{t}^{\prime}\right)$.

\section{Relations Between Bars And Edges}

Take a bar $B, h(B)<\infty$. Take $\beta \in \beta_{\phi} \in B$. Define $\zeta_{B}(y)$ to be $\beta(y)$ with all terms $y^{e}$ deleted, $e \geq h(B)$. Clearly, $\zeta_{B}(y)$ depends only on $B$, not on the choices of $\beta, \beta_{\phi}$.

Take an indeterminate $z$, and write

$$
\phi\left(\zeta_{B}(y)+z y^{h(B)}, y\right):=P_{B}(z) y^{L(B)}+\cdots, P_{B}(z) \not \equiv 0,
$$

where $L(B)$ was defined in (2.6) . We call $P_{B}(z)$ the associated polynomial of $B$.

Let $Z\left(P_{B}\right)$ denote the zero set of $P_{B}(z)$. Using the canonical coordinates, we can identify $B$ with $\mathbb{C}-Z\left(P_{B}\right)$. Hence $\bar{B}$, the metric space completion of $B$, is a copy of $\mathbb{C}$; and

$$
\operatorname{val}_{\phi}\left(\zeta_{B}(y)+z y^{h(B)}\right)=\left(P_{B}(z), L(B)\right) \in \mathcal{V}, \quad z \notin Z\left(P_{B}\right) .
$$

Take $\alpha \in \mathbb{M}_{1}$. If $\alpha(y)=\zeta_{B}(y)+a y^{h(B)}+\cdots, a \in \mathbb{C}$, we say $\bar{B}$ is a support of $\alpha$; a is the $\bar{B}$-coordinate of $\alpha$, and also of $\alpha_{\phi}$. Observe that $\alpha_{\phi} \in B$ iff $a \notin Z\left(P_{B}\right)$.

Notation 6.1. Write $\alpha \perp \bar{B}$ if $\alpha$ is supported by $\bar{B}$; Supp $(\alpha):=\{\bar{B} \mid \alpha \perp \bar{B}\}$.

Let $\alpha$ be given. We now define $\mathcal{N} \mathcal{P}_{\text {ext }}(\phi, \alpha)$ by adding "vertex edges" to $\mathcal{N} \mathcal{P}(\phi, \alpha)$.

Take a vertex $V_{i}=\left(m_{i}, q_{i}\right)$ of $\mathcal{N} \mathcal{P}(\phi, \alpha), m_{i} \geq 1$, representing a term $c y^{q_{i}} \xi^{m_{i}}$ in (2.3), where $c \neq 0$. Take $h \in \mathbb{Q}^{+}, \tan \theta_{i-1}<h<\tan \theta_{i}$. Let

$$
E(h):=\left(V_{i}, h\right), \quad P_{E(h)}(z):=c z^{m_{i}} .
$$

We call $E(h)$ a vertex edge and $P_{E(h)}(z)$ the associated polynomial. The height, or co-slope, of $E(h)$ is, by definition, $h(E(h)):=h$.

Let $\mathcal{N} \mathcal{P}_{\text {ext }}(\phi, \alpha)$ denote the edges $\left\{E_{0}, \ldots, E_{l-1}\right\}$ of $\mathcal{N} \mathcal{P}(\phi, \alpha)$ plus the vertex edges.

Convention 6.2. For an edge $E$ of $\mathcal{N} \mathcal{P}(\phi, \alpha), h:=h(E)<\infty$, we also write $E$ as $E(h)$.

Now we define

$$
\iota: \operatorname{Supp}(\alpha) \rightarrow \mathcal{N} \mathcal{P}_{\text {ext }}(\phi, \alpha), \quad \bar{B} \mapsto \iota(\bar{B}),
$$

where $\iota(\bar{B})$ is the unique edge of height (co-slope) $h(\iota(\bar{B}))=h(B)$. This is a bijection.

Take $\bar{B} \in \operatorname{Supp}(\alpha), h:=h(B)$. Let $c$ be the largest constant such that $\mathcal{N} \mathcal{P}(\phi, \alpha)$ is bounded below by the line $\mathcal{L}(h): u+v / h=c$. Let $i$ be the smallest integer such that 
$h \leq \tan \theta_{E_{i}}$. If $h=\tan \theta_{E_{i}}$, all dots on $E_{i}$ lie on $\mathcal{L}(h)$. If $h<\tan \theta_{E_{i}}, V_{i}$ is the only vertex lying on $\mathcal{L}(h)$. In either case, we define $\iota(\bar{B}):=E(h)$. See Fig, \$11.

The corresponding associated polynomials differ merely by a translation:

$$
P_{B}(z)=P_{\iota(\bar{B})}(z-a), \quad a \text { the } \bar{B} \text {-coordinate of } \alpha \text {. }
$$

\section{Proof Of The Morse Stability Theorem over $\mathbb{F}$}

Give $B, h(B)<\infty$. Take $\zeta \in Z(\phi), \zeta \perp \bar{B}$. Let $\zeta_{t}$ be the deformation of $\zeta$ in Corollary 5.6. Define $B_{t}$ to be the unique bar such that $h\left(B_{t}\right)=h(B), \zeta_{t} \perp \bar{B}_{t}$.

If $\zeta^{\prime} \in Z(\phi)$ and $\zeta^{\prime} \perp \bar{B}$, then $O_{y}\left(\zeta_{t}-\zeta_{t}^{\prime}\right)=O_{y}\left(\zeta-\zeta^{\prime}\right) \geq h(B)$. Hence $\operatorname{Supp}\left(\zeta_{t}\right), \operatorname{Supp}\left(\zeta_{t}^{\prime}\right)$ have the same set of bars of height $\leq h(B)$.

It follows that $B_{t}$ is well-defined (independent of the choice of $\zeta$ ),

$$
\mathcal{D}_{\text {bar }}: B \operatorname{sp}\left(\mathbb{M}_{1, \phi}\right) \times I_{\mathbb{F}} \rightarrow B \operatorname{sp}\left(\mathbb{M}_{1} \times_{\Phi} I_{\mathbb{F}}\right), \quad(B, t) \mapsto\left(B_{t}, t\right),
$$

is a homeomorphism, $h\left(B_{t}\right)=h(B)$.

Lemma 7.1. The family $\left\{P_{B_{t}}(z)\right\}$ is Morse stable (in the sense of Definition 5.4).

Proof. In $\mathcal{N} \mathcal{P}(\phi, \zeta)$, the left vertex of $E_{\text {top }}$ is $\left(m_{l}, q_{l}\right), m_{l} \geq 1$, where $m_{l}$ is the multiplicity of $\zeta($ as a root of $\phi)$. Therefore $P_{\text {top }}(0)=0, \operatorname{deg} P_{\text {top }}(z) \geq 2, P_{\text {top }}(z)$ is not a monomial.

Hence there exists $c, P_{\text {top }}^{\prime}(c)=0 \neq P_{\text {top }}(c)$. By Theorem [5.3, there exists $\gamma_{\phi} \in C\left(v a l_{\phi}\right)$, whose $\bar{B}$-coordinate is $c, B:=\iota^{-1}\left(E_{t o p}\right)$. Then $c$ has a deformation $c_{t}, c_{0}=c$,

$$
\tau_{t}\left(\gamma_{\phi}\right)(y)=\zeta_{B}(y)+c_{t} y^{h(B)}, \quad \mathcal{N} \mathcal{P}\left(\phi, \gamma_{\phi}\right)=\mathcal{N} \mathcal{P}\left(\phi_{t}, \tau_{t}\left(\gamma_{\phi}\right)\right)
$$

Let us compare the edge $E_{i}\left(\zeta_{t}\right)$ of $\mathcal{N} \mathcal{P}\left(\phi_{t}, \zeta_{t}\right)$ with $E_{i}\left(\tau_{t}\left(\gamma_{\phi}\right)\right)$ of $\mathcal{N} \mathcal{P}\left(\phi_{t}, \tau_{t}\left(\gamma_{\phi}\right)\right)$.

If $i \leq l-2$, then we clearly have $E_{i}\left(\zeta_{t}\right) \equiv E_{i}\left(\tau_{t}\left(\gamma_{\phi}\right)\right)$. Hence $P_{E_{i}\left(\zeta_{t}\right)}(z)=P_{E_{i}\left(\tau_{t}\left(\gamma_{\phi}\right)\right)}(z)$.

By the Fundamental Lemma, $\left\{P_{E_{i}\left(\tau_{t}\left(\gamma_{\phi}\right)\right)}(z)\right\}$ is Morse stable. Hence if $\bar{B}^{\prime} \in \operatorname{Supp}(\zeta)$ and $h\left(B^{\prime}\right)<\tan \theta_{\text {top }}$, then $\left\{P_{B_{t}^{\prime}}(z)\right\}$ is Morse stable.

As for the top edges $E_{t o p}\left(\zeta_{t}\right), E_{t o p}\left(\tau_{t}\left(\gamma_{\phi}\right)\right)$, their associated polynomials differ merely by a translation $z \mapsto z+c_{t}-a_{t}$, where $a_{t}$ is the $\bar{B}$-coordinate of $\zeta_{t}$. The stability of the latter implies that of the former.

Finally, if $h\left(B^{\prime \prime}\right)>\tan \theta_{t o p}$, then $P_{B_{t}^{\prime \prime}}(z)$ is a monomial, hence Morse stable.

Take $B \in B \operatorname{sp}\left(\mathbb{M}_{1, \phi}\right)$, and deformation $B_{t}$. Recall that $\bar{B}=\bar{B}_{t}=\mathbb{C}$. Applying the classical Morse Stability Theorem (\$10) to $\left\{P_{B_{t}}\right\}$, we have homeomorphisms $D_{t}, d_{t}$ such that

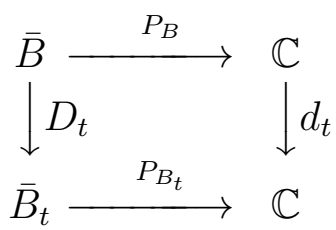

is commutative, where $D_{t}$ preserves the critical points and zeros $\left(d_{t}(0)=0\right)$.

Given $\alpha_{\phi} \in B$, with $\bar{B}$-coordinate $a$. Take $\alpha_{\phi, t} \in B_{t}$ whose $\bar{B}_{t}$-coordinate is $D_{t}(a)$ :

$$
\alpha_{\phi}(y)=\zeta_{B}(y)+a y^{h(B)}, \alpha_{\phi, t}(y)=\zeta_{B_{t}}(y)+D_{t}(a) y^{h(B)} \text {. }
$$


Thus, we have a homeomorphism:

$$
\mathcal{D}_{\Phi}: \mathbb{M}_{1, \phi} \times I_{\mathbb{F}} \rightarrow \mathbb{M}_{1} \times_{\Phi} I_{\mathbb{F}}, \quad\left(\alpha_{\phi}, t\right) \mapsto\left(\alpha_{\phi, t}, t\right) .
$$

Next, take $(u, L) \in \mathcal{V}, B \in B \operatorname{sp}\left(\mathbb{M}_{1, \phi}\right), L=L(B)(<\infty)$. We define

$$
\left.\mathcal{D}_{\mathcal{V}}((u, L), B, t)\right):=\left(\left(d_{t}(u), L\right), B_{t}, t\right) .
$$

If $h(B)=\infty$, then $B=\{\zeta\}, \zeta \in Z(\phi)$. We define

$$
\mathcal{D}_{\mathcal{V}}\left(0_{\mathcal{V}},\{\zeta\}, t\right):=\left(0_{\mathcal{V}},\left\{\zeta_{t}\right\}, t\right)
$$

We then have $\mathcal{D}_{\mathcal{V}} \circ V a l_{\phi}=V a l_{\Phi} \circ \mathcal{D}_{\Phi}$.

It remains to show that $\mathcal{D}_{\Phi}, \mathcal{D}_{\mathcal{V}}$ preserve the structures.

Take $\mu_{\phi}$. Take $\zeta \in Z(\phi)$ such that $h\left(\mu_{\phi}\right)=O\left(\mu_{\phi}-\zeta\right)$. Then

$$
h\left(\mu_{\phi}\right)=O\left(\mu_{\phi}-\zeta\right)=O\left(\mu_{\phi, t}-\zeta_{t}\right)=h\left(\mu_{\phi, t}\right) .
$$

Next, $\chi_{\text {puis }}\left(\mu_{\phi}\right)$ and $\chi_{\text {puis }}\left(\mu_{\phi, t}\right)$ can be expressed in terms of the co-slopes of the edges of $\mathcal{N} \mathcal{P}(\phi, \zeta)=\mathcal{N} \mathcal{P}\left(\phi_{t}, \zeta_{t}\right)$. Hence $\chi_{\text {puis }}\left(\mu_{\phi}\right)=\chi_{\text {puis }}\left(\mu_{\phi, t}\right)$.

As for the contact order, first suppose $\mu_{\phi} \sim_{\text {bar }} \nu_{\phi}$. Then there exists $\zeta \in Z(\phi)$,

$$
h\left(\mu_{\phi}\right)=h\left(\nu_{\phi}\right)=O\left(\mu_{\phi}-\zeta\right)=O\left(\nu_{\phi}-\zeta\right),
$$

which remain valid when the parameter $t$ is added. Hence $\mathcal{C}_{\text {ord }}\left(\mu_{\phi}, \nu_{\phi}\right)=\mathcal{C}_{\text {ord }}\left(\mu_{\phi, t}, \nu_{\phi, t}\right)$.

Now suppose $B\left(\mu_{\phi}\right) \neq B\left(\nu_{\phi}\right)$. Take $\zeta \perp \bar{B}\left(\mu_{\phi}\right), \zeta^{\prime} \perp \bar{B}\left(\nu_{\phi}\right)$. Then

$$
\mathcal{C}_{\text {ord }}\left(\mu_{\phi}, \nu_{\phi}\right)=O\left(\zeta-\zeta^{\prime}\right)=O\left(\zeta_{t}-\zeta_{t}^{\prime}\right)=\mathcal{C}_{\text {ord }}\left(\mu_{\phi, t}, \nu_{\phi, t}\right) .
$$

\section{The Trivialization Vector Field}

When a coordinate system $\left(z_{1}, \ldots, z_{n}\right)$ of $\mathbb{C}^{n}$ is chosen, we use $\left\{\frac{\partial}{\partial z_{1}}, \ldots, \frac{\partial}{\partial z_{n}}\right\}$ to denote the standard orthonormal basis, with hermitian product

$$
<\sum a_{i} \frac{\partial}{\partial z_{i}}, \sum b_{i} \frac{\partial}{\partial z_{i}}>=\sum a_{i} \bar{b}_{i} \quad\left(\bar{b}_{i} \text { the complex conjugate of } b_{i}\right) .
$$

For a holomorphic function $h\left(z_{1}, \ldots, z_{n}\right)$, the gradient of $h$ ([17], p.33) is

$$
\operatorname{Grad} h:=\sum \overline{\frac{\partial h}{\partial z_{i}}} \frac{\partial}{\partial z_{i}} .
$$

Let $f(x, y), F(x, y, t), \phi_{t}, \Phi$, be as in $\$ 3$. To prove the Equi-singular Deformation Theorem, we use a vector field $\overrightarrow{\mathcal{F}}(x, y, t)$ which is defined in two steps, following Ehresmann's idea ([16]), where $(x, y, t) \in \mathcal{N}, \mathcal{N}$ a sufficiently small neighborhood of $\{0\} \times I_{\mathbb{C}}$ in $\mathbb{C}^{2} \times I_{\mathbb{C}}$.

Step One. Take $\gamma_{\phi} \in C\left(v_{a l}\right)$, with deformation $\gamma(y, t):=\tau_{t}\left(\gamma_{\phi}\right)(y)$ as in (2.8). In this step we assume $\gamma(y, t)$ is a holomorphic function in $(y, t), \gamma(0, t) \equiv 0$.

The curve-germ defined by $x=\gamma(y, t)$ is smooth, $t \in I_{\mathbb{C}}$.

We define $\overrightarrow{\mathcal{F}}_{\gamma}(x, y, t)$ as follows. The coordinate transformation

$$
\mathcal{D}:(x, y, t) \mapsto\left(x_{\gamma}, y_{\gamma}, t_{\gamma}\right):=(x-\gamma(y, t), y, t)
$$


is holomorphic, $\mathcal{D}^{-1}$ transforms $F(x, y, t)$ to

$$
F^{(\gamma)}\left(x_{\gamma}, y_{\gamma}, t_{\gamma}\right):=F\left(x_{\gamma}+\gamma\left(y_{\gamma}, t_{\gamma}\right), y_{\gamma}, t_{\gamma}\right)
$$

Convention 8.1. We shall use $F_{x_{\gamma}}, F_{y_{\gamma}}, F_{t_{\gamma}}$ to denote the partial derivatives of $F^{(\gamma)}$. The notations are simpler, but cause no confusion.

Now, consider the vector field

$$
\vec{V}\left(x_{\gamma}, y_{\gamma}, t_{\gamma}\right):=-A\left(x_{\gamma}, y_{\gamma}, t_{\gamma}\right) x_{\gamma} \frac{\partial}{\partial x_{\gamma}}-B\left(x_{\gamma}, y_{\gamma}, t_{\gamma}\right) y_{\gamma} \frac{\partial}{\partial y_{\gamma}}+\frac{\partial}{\partial t_{\gamma}},
$$

where

$$
A:=\frac{\bar{x}_{\gamma} \bar{F}_{x_{\gamma}} F_{t_{\gamma}}}{\left|x_{\gamma} F_{x_{\gamma}}\right|^{2}+\left|y_{\gamma} F_{y_{\gamma}}\right|^{2}}, \quad B:=\frac{\bar{y}_{\gamma} \bar{F}_{y_{\gamma}} F_{t_{\gamma}}}{\left|x_{\gamma} F_{x_{\gamma}}\right|^{2}+\left|y_{\gamma} F_{y_{\gamma}}\right|^{2}}
$$

and, by the Chain Rule,

$$
\frac{\partial}{\partial x_{\gamma}}=\frac{\partial}{\partial x}, \quad \frac{\partial}{\partial y_{\gamma}}=\frac{\partial}{\partial y}+\frac{\partial \gamma}{\partial y} \frac{\partial}{\partial x}, \quad \frac{\partial}{\partial t_{\gamma}}=\frac{\partial}{\partial t}+\frac{\partial \gamma}{\partial t} \frac{\partial}{\partial x}
$$

The coefficients $A, B$ are chosen so that $\langle\vec{V}, \operatorname{Grad} F\rangle=0$. Hence $\vec{V}$ is tangent to the level surfaces $F=$ const. (Here Grad $F:=\bar{F}_{x_{\gamma}} \frac{\partial}{\partial x_{\gamma}}+\bar{F}_{y_{\gamma}} \frac{\partial}{\partial y_{\gamma}}+\bar{F}_{t_{\gamma}} \frac{\partial}{\partial t_{\gamma}}$.)

We have not defined $\vec{V}$ when $x_{\gamma} F_{x_{\gamma}}=y_{\gamma} F_{y_{\gamma}}=0$. This we shall do in 99 ,

Using (8.3) we can express $\vec{V}$ as a vector field in the $(x, y, t)$-space:

$$
\overrightarrow{\mathcal{F}}_{\gamma}(x, y, t):=(d \mathcal{D})^{-1}(\vec{V}),
$$

which is tangent to $F=$ const. Each trajectory (integral curve) lies on a single level surface.

When $x_{\gamma}=0$, the $\partial / \partial x_{\gamma}$ component of $\vec{V}$ vanishes, hence the flow generated by $\vec{V}$ carries the $y_{\gamma}$-axis to itself (but not necessarily point-wise fixed). The flow generated by $\overrightarrow{\mathcal{F}}_{\gamma}$, in the $(x, y, t)$-space, carries the curve-germ $\pi_{*}(\gamma(y, 0))$ to $\pi_{*}(\gamma(y, t))$ at time $t$.

Step Two. We are to define $\overrightarrow{\mathcal{F}}(x, y, t)$. Take $\gamma_{j, \phi} \in C\left(v a l_{\phi}\right)$ in (2.7). Take the deformation $\tau_{t}\left(\gamma_{j, \phi}\right)$ in (2.8). Write the canonical coordinate simply as

$$
\gamma_{j}(y, t):=\tau_{t}\left(\gamma_{j, \phi}\right)(y), \quad 1 \leq j \leq p .
$$

Take an integer $N$ divisible by every $m_{\text {puis }}\left(\gamma_{j, \phi}\right)$, for instance, $N:=\prod_{j} m_{p u i s}\left(\gamma_{j, \phi}\right)$.

Consider the substitution map

$$
S_{X Y}:(X, Y, T) \mapsto(x, y, t):=\left(X, Y^{N}, T\right),
$$

and the coordinate transformation

$$
X_{j}:=X-\Gamma_{j}(Y, T), \quad Y_{j}:=Y, \quad T_{j}:=T,
$$

where $\Gamma_{j}(Y, T):=\gamma_{j}\left(Y^{N}, T\right)$ is holomorphic. Like (8.1), we write

$$
F^{(j)}\left(X_{j}, Y_{j}, T_{j}\right):=F\left(X_{j}+\Gamma_{j}, Y_{j}^{N}, T_{j}\right), \quad 1 \leq j \leq p .
$$

Of course $X_{j}=0$ is smooth, and is mapped by $S_{X Y}$ to $\pi_{*}\left(\gamma_{j, \phi}\right), 1 \leq j \leq p$. (The latter may not be mutually distinct: if $\gamma_{1, \phi}(y), \gamma_{2, \phi}(y)$ are conjugates, then $\pi_{*}\left(\gamma_{1, \phi}\right)=\pi_{*}\left(\gamma_{2, \phi}\right)$.) 
Therefore, for each $j, \overrightarrow{\mathcal{F}}_{\Gamma_{j}}(X, Y, T)$ is defined as in (8.4), being the vector field

$$
\vec{V}_{j}\left(X_{j}, Y_{j}, T_{j}\right):=-A_{j}\left(X_{j}, Y_{j}, T_{j}\right) X_{j} \frac{\partial}{\partial X_{j}}-B_{j}\left(X_{j}, Y_{j}, T_{j}\right) Y_{j} \frac{\partial}{\partial Y_{j}}+\frac{\partial}{\partial T_{j}}
$$

expressed in terms of $(X, Y, T)$, where, as in (8.2), with Convention 8.1,

$$
A_{j}:=\frac{\bar{X}_{j} \bar{F}_{X_{j}} F_{T_{j}}}{\left|X_{j} F_{X_{j}}\right|^{2}+\left|Y_{j} F_{Y_{j}}\right|^{2}}, \quad B_{j}:=\frac{\bar{Y}_{j} \bar{F}_{Y_{j}} F_{T_{j}}}{\left|X_{j} F_{X_{j}}\right|^{2}+\left|Y_{j} F_{Y_{j}}\right|^{2}} .
$$

Let us write $\hat{X}_{k}:=X_{1} \cdots X_{k-1} \cdot X_{k+1} \cdots X_{p}$, and, for $(X, Y) \neq(0,0)$, define

$$
\mathcal{P}_{k}:=\frac{\left|\hat{X}_{k}\right|^{2}}{\left|\hat{X}_{1}\right|^{2}+\cdots+\left|\hat{X}_{p}\right|^{2}}, \quad 1 \leq k \leq p .
$$

We call $\left\{\mathcal{P}_{k}\right\}$ a partition of unity, for we have

$$
\sum \mathcal{P}_{k}=1, \quad \mathcal{P}_{k}=1 \text { when } X_{k}=0, \quad \mathcal{P}_{k}=0 \text { when } X_{j}=0, j \neq k .
$$

The $\mathcal{P}_{k}$ 's are real analytic at every $(X, Y) \neq(0,0)$ in the sense of Convention 1.1 .

Now we use the $\mathcal{P}_{k}$ 's to "patch up" the vectors $\overrightarrow{\mathcal{F}}_{\Gamma_{j}}$ :

$$
\vec{v}(X, Y, T):=\mathcal{P}_{1} \vec{V}_{1}+\cdots+\mathcal{P}_{p} \vec{V}_{p} \quad(\text { all expressed in } X, Y, T),
$$

and, using the differential $d S_{X Y}$ of the substitution map $S_{X Y}$, define

$$
\overrightarrow{\mathcal{F}}(x, y, t):=d S_{X Y}(\vec{v}) .
$$

We must show $\overrightarrow{\mathcal{F}}$ is well-defined, since $S_{X Y}$ is a many-to-one mapping.

Let $\theta:=e^{2 \pi \sqrt{-1} / N}$. A conjugation $y^{1 / N} \mapsto \theta^{j} y^{1 / N}$ permutes the $\gamma_{k}$ 's, the $X_{k}$ 's and the $\mathcal{P}_{k}$ 's. Hence $\vec{v}(X, Y, T)$ is invariant under these conjugations. It follows that $\overrightarrow{\mathcal{F}}$ is well-defined.

We shall show, in 99 , that $\overrightarrow{\mathcal{F}}$ can be extended continuously throughout $\mathcal{N}$, so that $\overrightarrow{\mathcal{F}}(x, y, t)$ is well-defined in $\mathcal{N}$, tangent to $F=$ const. Because of (8.8), the flow generated by $\overrightarrow{\mathcal{F}}$ carries the curve-germ $\pi_{*}\left(\gamma_{j}(y, 0)\right)$ to $\pi_{*}\left(\gamma_{j}(y, t)\right)$ at time $t, 1 \leq j \leq p$.

Attention 8.2. It is important to point out what the above does not say.

Take $\gamma_{j, \phi}$. Of course there exists $\gamma_{j}(y):=\gamma_{j, \phi}(y)+\cdots$ such that $F_{x}\left(\gamma_{j}(y), y, 0\right)=0$. Hence $F_{x}(x, y, 0)=0$ on the curve-germ $\Delta:=\pi_{*}\left(\gamma_{j}(y)\right) ; \Delta$ is called a "polar" of $F(x, y, 0)$.

Note that $\mathcal{C}_{\text {ord }}\left(\Delta, \pi_{*}\left(\gamma_{j, \phi}\right)\right)>O_{y}\left(\gamma_{j, \phi}\right)$, and, in general, $\Delta \neq \pi_{*}\left(\gamma_{j, \phi}\right)$. Following the flow, $\Delta$ reaches $\Delta_{t}$ at time $t$. The above does not say $\Delta_{t}$ is necessarily a polar of $F(x, y, t)$.

For example, the Pham family $P(x, y, t)$ in (10.1) has only one polar when $t=0$, but two polars when $t \neq 0$. We have no idea whether the polar at $t=0$ will flow to one of the two polars, or more likely to neither. In the blurred space $\mathbb{C}_{* / P_{t}}$, however, there is a unique critical point for each $t \in I_{\mathbb{C}}$; they constitute a single orbit of $\left\{\eta_{t}\right\}$.

A critical point is an equivalence class in $\mathbb{C}_{*}$ containing at least one polar. We see the critical point at all time $t$, but cannot keep track of the polars. (This is like the Arakawa in Japan, a river which flows by Saitama University. We see the river bed, but cannot predict the position of the flow, whence, literally, the name "Arakawa"-Wild River.) 


\section{Proof of the Equi-Singular Deformation Theorem}

Recall that $F$ is mini-regular in $x$. In a sector $|y| \leq \epsilon|x|$, the behavior of $F$ is dominated by $x^{m}, m:=O(F)$. Hence there is nothing to worry about in this sector.

We shall henceforth restrict our attention to a sector $|x|<K|y|, K$ sufficiently large.

Notation 9.1. In this section we write $g \lesssim h$ if $g \leq C h, C>0$ a constant; $g \approx h$ means $g \lesssim h \lesssim g ;$ and $g \ll h$ means $g / h \rightarrow 0$.

Next we show how $\vec{v}$ in $(\underline{8.9})$ and $\overrightarrow{\mathcal{F}}$ in $(\underline{8.10})$ generate homeomorphisms.

The following is a parameterized version of the Proposition in [18, p.347.

Lemma 9.2. $\operatorname{For} F^{(j)}\left(X_{j}, Y_{j}, T_{j}\right):=F\left(X_{j}+\Gamma_{j}\left(Y_{j}, T_{j}\right), Y_{j}^{N}, T_{j}\right)$, we have

$$
\left|F_{T_{j}}\right| \lesssim\left|X_{j} F_{X_{j}}\right|+\left|Y_{j} F_{Y_{j}}\right|, \quad\left(X_{j}, Y_{j}, T_{j}\right) \in S_{X Y}^{-1}(\mathcal{N}),
$$

where $1 \leq j \leq p, \mathcal{N}$ as in $\S$. (We use Convention (8.1) : $F_{X_{j}}:=F_{X_{j}}^{(j)}$, etc..)

We can extend $A_{j}, B_{j}$ real analytically to $S_{X Y}^{-1}(\mathcal{N})-\{0\} \times I_{\mathbb{C}}$, where they are bounded.

Define $\overrightarrow{\mathcal{F}}(0,0, t):=\frac{\partial}{\partial t}$. Then $\overrightarrow{\mathcal{F}}$ is continuous on $\mathcal{N}$,

$$
\|\overrightarrow{\mathcal{F}}(x, y, t)-\partial / \partial t\| \lesssim|x|+|y|, \quad(x, y, t) \in \mathcal{N}
$$

and $\overrightarrow{\mathcal{F}}$ is real analytic in $\mathcal{N}-\{0\} \times I_{\mathbb{C}}$.

It follows that a trajectory of $\overrightarrow{\mathcal{F}}$, with initial point outside $I_{\mathbb{C}}$, will never reach $I_{\mathbb{C}}$.

The flow generated by $\overrightarrow{\mathcal{F}}$ carries $\pi_{*}\left(\gamma_{j, \phi}\right)$ to $\pi_{*}\left(\tau_{t}\left(\gamma_{j, \phi}\right)\right), 1 \leq j \leq p$.

Proof. We use the Curve Selection Lemma to prove (9.1). Let $\rho(s)$ be a given analytic arc. It suffices to show that (9.1) holds along $\rho$. We can assume $\rho(0)=0$.

Take $\varepsilon^{\prime}>0$, sufficiently small. For each pair $\Gamma_{k}, \Gamma_{s}$ (in $\left.\S\right]$ ), let $d_{k s}:=O_{Y}\left(\Gamma_{k}-\Gamma_{s}\right)$.

Define a horn neighborhood of $\Gamma_{k}$ of order $d_{k s}$ by

$$
H_{d_{k s}}\left(\Gamma_{k}\right):=\left\{(X, Y, T)|| X-\left.\Gamma_{k}(Y, T)\left|<\varepsilon^{\prime}\right| Y\right|^{d_{k s}}\right\} .
$$

This is a sub-analytic set, hence either $\operatorname{Im}(\rho)-\{0\} \subset H_{d_{k s}}\left(\Gamma_{k}\right)$ or $\operatorname{Im}(\rho) \cap H_{d_{k s}}\left(\Gamma_{k}\right)=\emptyset$.

Let us first consider the case where $\operatorname{Im}(\rho)-\{0\}$ is contained in at least one of the horn neighborhoods. In this case, by permuting the indices, if necessary, we can assume $H_{d_{12}}\left(\Gamma_{1}\right)$ is the smallest horn neighborhood containing $\operatorname{Im}(\rho)-\{0\}$.

We then work in the coordinate system $\left(X_{1}, Y_{1}, T_{1}\right)$, writing $\rho(s)=\left(X_{1}(s), Y_{1}(s), T_{1}(s)\right)$.

Let $\rho_{\pi}(x):=\left(X_{1}(s), Y_{1}(s)\right)$. We show (9.1) holds on the surface $\operatorname{Im}\left(\rho_{\pi}\right) \times I_{\mathbb{C}}$.

Let us write

$$
F^{(1)}\left(X_{1}, Y_{1}, T_{1}\right):=F_{0}^{(1)}\left(X_{1}, Y_{1}\right)+P\left(X_{1}, Y_{1}, T_{1}\right), \quad P\left(X_{1}, Y_{1}, 0\right) \equiv 0 .
$$

By the Fundamental Lemma, $\mathcal{N} \mathcal{P}\left(F^{(1)}, 0\right)=\mathcal{N} \mathcal{P}\left(F_{0}^{(1)}, 0\right)$, all dots of $P\left(X_{1}, Y_{1}, T_{1}\right)$ lie on or above this polygon. (Newton Polygon at 0 is Newton Polygon in the usual sense.)

Convention 9.3. Consider the vertex $V_{l}=\left(m_{l}, q_{l}\right)$. Suppose $m_{l}=0$ and $h>\tan \theta_{\text {top }}$. In this section, we call the pair $E(h):=\left(V_{l}, h\right)$ also a vertex edge, with co-slope $h$. 
First, suppose $\operatorname{Im}(\rho)$ is not contained in $X_{1}=0$. We write the coordinate of $\rho_{\pi, \mathbb{C}}$ as

$$
\rho_{\pi}\left(Y_{1}\right):=r Y_{1}^{h}+\cdots, \quad r \neq 0, \quad d_{12} \leq h<\infty .
$$

And let $E:=E(h)$ denote the unique (possibly vertex) edge with co-slope $h$.

We first prove (9.1) for $j=1$.

Assume $E:=E(h)$ is a proper edge (i.e., not a vertex edge).

In the first place we must have $P_{E}^{\prime}(r) \neq 0$. For if $P_{E}^{\prime}(r)=0$, then, by Theorem 5.3, there would exist $\Gamma_{j}, j \geq 2$, of the form $\Gamma_{j}=r Y_{1}^{h}+\cdots$. Then $H_{d_{12}}\left(\Gamma_{1}\right)$ would not be the smallest horn neighbourhood containing $\operatorname{Im}(\rho)$, a contradiction.

Now we collect the monomial terms of $F^{(1)}$ along $E$ :

$$
W\left(X_{1}, Y_{1}, T_{1}\right):=\sum a_{p q}(t) X_{1}^{p} Y_{1}^{q}, \quad(p, q) \in E,
$$

which is a weighted form, $W(z, 1, t)=P_{E}(z)$.

Take $u \neq 0$. Let $t$ be fixed. By Euler's Theorem, $X_{1}-u Y_{1}^{h}$ is a common factor of $X_{1} W_{X_{1}}$ and $Y_{1} W_{Y_{1}}$ iff $\left(X_{1}-u Y_{1}^{h}\right)^{2}$ divides $W\left(X_{1}, Y_{1}, T_{1}\right)$, i.e., $P_{E}(u)=P_{E}^{\prime}(u)=0$.

Hence, if $\left(P_{E}(r), P_{E}^{\prime}(r)\right) \neq(0,0)$, then, along $\rho_{\pi}$,

$$
O_{Y_{1}}\left(\left|X_{1} W_{X_{1}}\right|+\left|Y_{1} W_{Y_{1}}\right|\right)=O_{Y_{1}}\left(\left|X_{1} F_{X_{1}}\right|+\left|Y_{1} F_{Y_{1}}\right|\right)=q_{E}+m_{E} h,
$$

where $\left(m_{E}, q_{E}\right)$ is any dot on $E$. It follows that

$$
L_{\left|X_{1} F_{X_{1}}\right|+\left|Y_{1} F_{Y_{1}}\right|}\left(\rho_{\pi}\right)=q_{E}+m_{E} h .
$$

All dots of $F_{T_{1}}$ lie on or above the line $\mathcal{L}(E), O_{Y_{1}}\left(F_{T_{1}}\right) \geq q_{E}+m_{E} h$, proving (9.1).

If $E=\left(V_{k}, h\right)$ is a vertex edge, then $\left|Y_{1} F_{Y_{1}}\right| \approx|r|^{m_{k}}\left|Y_{1}\right|^{q_{k}+m_{k} h} \gtrsim\left|F_{T_{1}}\right|$. Again (9.1) holds.

Next we prove (9.1) for the case $j \geq 2$. The coordinate systems are related by

$$
X_{j}=X_{1}-\delta_{j}\left(Y_{1}, T_{1}\right), \quad Y_{j}=Y_{1}, \quad T_{j}=T_{1},
$$

where $\delta_{j}:=\Gamma_{j}-\Gamma_{1}\left(\delta_{1} \equiv 0\right)$. Let us write

$$
\delta_{j}\left(Y_{1}, T_{1}\right):=c_{j}\left(T_{1}\right) Y_{1}^{O\left(\delta_{j}\right)}+\cdots, \quad c_{j}(0) \neq 0, \quad 2 \leq j \leq p .
$$

Note that

and, by the Chain Rule,

$$
\left|Y_{1} \frac{\partial \delta_{j}}{\partial Y_{1}}\right| \approx\left|\delta_{j}\right|, \quad\left|\frac{\partial \delta_{j}}{\partial T_{1}}\right| \lesssim\left|\delta_{j}\right|
$$

$$
X_{j} F_{X_{j}}=\left(X_{1}-\delta_{j}\right) F_{X_{1}}, Y_{j} F_{Y_{j}}=Y_{1} F_{Y_{1}}+Y_{1} \frac{\partial \delta_{j}}{\partial Y_{1}} F_{X_{1}}, F_{T_{j}}=F_{T_{1}}+\frac{\partial \delta_{j}}{\partial T_{1}} F_{X_{1}} .
$$

Now, if $h \geq O\left(\delta_{j}\right)$, then, along $\rho_{\pi},\left|X_{1}-\delta_{j}\right| \approx\left|\delta_{j}\right|$. Hence (9.1) follows from

$$
\left|X_{j} F_{X_{j}}\right|+\left|Y_{j} F_{Y_{j}}\right| \approx\left|\delta_{j} F_{X_{1}}\right|+\left|Y_{1} F_{Y_{1}}\right|, \quad\left|F_{T_{j}}\right| \lesssim\left|F_{T_{1}}\right|+\left|\delta_{j} F_{X_{1}}\right|,
$$

and (9.1) when $j=1$.

Suppose $h<O\left(\delta_{j}\right)$. Then $\left|X_{1}-\delta_{j}\right| \approx\left|X_{1}\right|$ along $\rho_{\pi}$. Again we have (9.1). 
Suppose $\operatorname{Im}(\rho)$ is contained in $X_{1}=0$. In this case $F$ is divisible by $X_{1}^{m_{l}}, F_{T_{1}}$ is divisible by $X_{1}^{k}, k \geq m_{l}$. Again, we have (9.1).

It remains to consider the case where no horn neighborhood contains $\operatorname{Im}(\rho)-\{0\}$. We again write $\rho_{\pi}$ as (9.5) where now $h<d_{j k} \forall j, k$. The same argument proves (9.1).

Next we show how $A_{j}, B_{j}$ can be extended across $S_{X Y}^{-1}(\mathcal{N})-\{0\} \times I_{\mathbb{C}}$.

Lemma 9.4. The zero set of the denominator of $A_{j}, B_{j}$ is

$$
\left\{(X, Y, T) \mid X_{j} F_{X_{j}}=Y_{j} F_{Y_{j}}=0\right\}=\left\{\begin{array}{l}
\bigcup\left\{X_{k}=0 \mid X_{k}^{2} \text { divides } F\right\} \\
\{0\} \text { if no such } X_{k} \text { exists. }
\end{array}\right.
$$

Proof. Recall that we work in the sector $|x|<K|y|$, hence $Y \neq 0$.

Suppose $X_{j}=F_{Y_{j}}=0$. In $\mathcal{N} \mathcal{P}\left(F^{(j)}, \Gamma_{j}\right)$, which is independent of $T_{j}$, we must have $m_{l} \geq 2$. Hence $F$ is divisible by $X_{j}^{2}$.

Let $\rho(s)$ be an analytic arc along which $F_{X_{j}}=F_{Y_{j}}=0$. Then $F_{X}=F_{Y}=0$ along $\rho$. As before, we choose $X_{1}$, define $\rho_{\pi}, F_{0}\left(X_{1}, Y_{1}\right)$, etc., as in (9.4), and then

$$
\frac{\partial F_{0}^{(1)}}{\partial X_{1}}=\frac{\partial F_{0}^{(1)}}{\partial Y_{1}}=0 \text { along } \rho_{\pi} \text {. }
$$

Hence $\operatorname{Im}\left(\rho_{\pi, \mathbb{C}}\right)$ must be $X_{1}=0\left(T_{1}=0\right), X_{1}^{2}$ divides $F_{0}$, hence also $F$.

We can now complete the proof of Lemma 9.2 .

The real meromorphic functions $A_{j}, B_{j}$ are bounded, by (9.1). Hence, if $n$ is the largest integer such that $X_{k}^{n}$ divides both $X_{j} F_{X_{j}}$ and $Y_{j} F_{Y_{j}}$, then $X_{k}^{n}$ must also divides $F_{T_{j}}$.

It follows that $A_{j}, B_{j}$ are defined and real analytic on $S_{X Y}^{-1}(\mathcal{N})-\{0\} \times I_{\mathbb{C}}$.

Hence $\overrightarrow{\mathcal{F}}(x, y, t)$, with $\overrightarrow{\mathcal{F}}(0,0, t):=\frac{\partial}{\partial t}$, is continuous in $\mathcal{N}$, satisfying (9.2), and is real analytic in $\mathcal{N}-\{0\} \times I_{\mathbb{C}}$.

Finally, $\mathcal{P}_{k} X_{k}=0$ along every $X_{j}=0$. Hence $\overrightarrow{\mathcal{F}}$ carries $\pi_{*}\left(\gamma_{j, \phi}\right)$ to $\pi_{*}\left(\tau_{t}\left(\gamma_{i, \phi}\right)\right)$.

Now, using a well-known argument ([12]), (9.2) implies that $\overrightarrow{\mathcal{F}}$ generates a homeomorphism $H$ having properties (3.1), (1) and (2) in the Equi-singular Deformation Theorem.

Next we prove $(3)$. Let $\rho(s)=(x(s), y(s))$ be a given analytic arc in the $(x, y)$-plane. Consider $\rho^{(N)}(s):=\left(x\left(s^{N}\right), y\left(s^{N}\right)\right)$. This arc can be lifted by $S_{X Y}$ to an analytic arc

$$
\rho_{X Y}(s):=(X(s), Y(s)), \quad S_{X Y} \circ \rho_{X Y}=\rho^{(N)},
$$

where $Y(s)$ is an integral power series in $s$ obtained by solving $Y^{N}=y\left(s^{N}\right)$. (Of course, there are $N$ liftings of $\rho^{(N)}$ to the $(X, Y)$-space; in general, however, we cannot lift $\rho$.)

Let us write $\Gamma_{j}:=\Gamma_{j}(Y, 0)$. By permuting the indices, if necessary, we can assume

$$
\begin{aligned}
h & :=\mathcal{C}_{\text {ord }}\left(\operatorname{Im}\left(\rho_{X Y, \mathbb{C}}\right), \operatorname{Im}\left(\Gamma_{1}\right)\right)=\cdots=\mathcal{C}_{\text {ord }}\left(\operatorname{Im}\left(\rho_{X Y, \mathbb{C}}\right), \operatorname{Im}\left(\Gamma_{r}\right)\right) \\
& >\mathcal{C}_{\text {ord }}\left(\operatorname{Im}\left(\rho_{X Y, \mathbb{C}}\right), \operatorname{Im}\left(\Gamma_{r+i}\right)\right), \quad 0<i \leq p-r,
\end{aligned}
$$

where $1 \leq r \leq p, h / N=\mathcal{C}_{\text {ord }}\left(\operatorname{Im}\left(\rho_{\mathbb{C}}\right), \pi_{*}\left(\gamma_{j, \phi}\right)\right), 1 \leq j \leq r$. 
There are now three cases to consider: (a) $h / N \leq h\left(\gamma_{1, \phi}\right), h<\infty$, (b) $h\left(\gamma_{1, \phi}\right)<h / N<\infty$, and (c) $h=\infty$, that is, $\operatorname{Im}\left(\rho_{\mathbb{C}}\right)=\pi_{*}\left(\gamma_{1, \phi}\right)$.

Case (c) is easy: $\operatorname{Im}\left(\rho_{X Y, \mathbb{C}}\right)$ is the $Y_{1}$-axis, along which $\vec{v}=\vec{V}_{1}$ is analytic, whence (3).

Consider case (a). Let $E:=E(h)$ be the unique edge in $\mathcal{N} \mathcal{P}_{\text {ext }}\left(F_{0}^{(1)}, 0\right)$ of co-slope $h$. ( Of course, $E$ can be a vertex edge $\left(V_{e}, h\right)$. In this case $m_{e}>0, P_{E}(z)$ is a monomial.)

As before, we work in the coordinate system $\left(X_{1}, Y_{1}, T_{1}\right)$.

Take a coordinate of $\operatorname{Im}\left(\rho_{X Y, \mathbb{C}}\right)$,

$$
\rho_{X Y}\left(Y_{1}\right):=u_{\rho} Y_{1}^{h}+\cdots, \quad u_{\rho} \neq 0 .
$$

A coordinate of $\operatorname{Im}\left(\rho_{\mathbb{C}}\right)$ is

$$
\rho(y)=\gamma_{1, \phi}(y)+\left[u_{\rho} y^{h / N}+\cdots\right] .
$$

As before, $P_{E}^{\prime}\left(u_{\rho}\right) \neq 0$. (Same argument: otherwise, (9.11) would fail.)

Now, let us first assume that $h$ is an integer, so that the substitution map

$$
S_{u v}:(u, v, t) \mapsto\left(X_{1}, Y_{1}, T_{1}\right):=\left(u v^{h}, v, t\right),
$$

is holomorphic, where

$$
X_{1} \frac{\partial}{\partial X_{1}}=u \frac{\partial}{\partial u}, \quad Y_{1} \frac{\partial}{\partial Y_{1}}=-h u \frac{\partial}{\partial u}+v \frac{\partial}{\partial v}, \quad \frac{\partial}{\partial T_{1}}=\frac{\partial}{\partial t} .
$$

We then define

$$
\vec{U}(u, v, t):=\left(d S_{u v}\right)^{-1}(\vec{v}) .
$$

Question 9.5. At which $(u, v, t)$ is $\vec{U}$ well-defined and real analytic?

To answer this, we need a careful analysis of the denominator of $A_{j}, B_{j}$ :

$$
D_{j}:=\left|X_{j} F_{X_{j}}\right|^{2}+\left|Y_{j} F_{Y_{j}}\right|^{2}, \quad 1 \leq j \leq p,
$$

and also that of $\mathcal{P}_{k}$, when the substitution $S_{u v}$ is made.

Lemma 9.6. Let $L(E):=q_{E}+m_{E} h,\left(m_{E}, q_{E}\right) \in E$. Then

$$
D_{j}\left(u v^{h}, v, t\right)=C_{j}(u, t) v^{2 L(E)}+\cdots, \quad 1 \leq j \leq r,
$$

where $C_{j}(u, t)$ is a polynomial in $u, \bar{u}$, coefficients in $t$.

For $u \neq 0, C_{j}(u, t)=0$ iff $P_{E}(u)=P_{E}^{\prime}(u)=0$. For any $u, P_{E}^{\prime}(u) \neq 0$ implies $C_{j}(u, t) \neq 0$.

(The coefficients of $P_{E}(u)$ are functions of $t$. To say $P_{E}(u)=P_{E}^{\prime}(u)=0$ means that when $t$ is fixed, $u$ is a multiple root of $P_{E}(z)$.)

Proof. Consider the weighted form $W\left(X_{1}, Y_{1}, T_{1}\right)$ in (9.6). Let

$$
w_{1}(z, t):=W_{X_{1}}(z, 1, t), \quad w_{2}(z, t):=W_{Y_{1}}(z, 1, t) .
$$

Take $j, 1 \leq j \leq r$. By (9.11), $O\left(\delta_{j}\right) \geq h, \delta_{j}$ being defined in (9.8). Let us write

$$
\hat{c}_{j}(t):= \begin{cases}c_{j}(t) & \text { if } O\left(\delta_{j}\right)=h, \\ 0 & \text { if } O\left(\delta_{j}\right)>h,\end{cases}
$$


where $c_{j}(t)$ is defined in (9.9). We claim that (9.13) holds if we take

$$
C_{j}(u, t):=\left|\left(u-\hat{c}_{j}(t)\right) w_{1}(u, t)\right|^{2}+\left|w_{2}(u, t)+h \hat{c}_{j}(t) w_{1}(u, t)\right|^{2},
$$

which is of course a polynomial in $u, \bar{u}$. Indeed, we have, as before,

$$
D_{j}=\left|\left(X_{1}-\delta_{j}\right) F_{X_{1}}\right|^{2}+\left|Y_{1} F_{Y_{1}}+Y_{1} \frac{\partial \delta_{j}}{\partial Y_{1}} F_{X_{1}}\right|^{2}
$$

whence the leading term of $D_{j}\left(u v^{h}, v, t\right)$ is the above $C_{j}(u, t)$.

The $\hat{c}_{i}(t)$ 's are roots of $w_{1}(z, t)=P_{E}^{\prime}(z)$. Hence $C_{j}(u, t)=0$ iff $w_{1}(u, t)=w_{2}(u, t)=0$.

For $u \neq 0, w_{1}=w_{2}=0$ iff $\left(X_{1}-u Y_{1}^{h}\right)^{2}$ divides $W\left(X_{1}, Y_{1}, T_{1}\right)$, i.e., $P_{E}(u)=P_{E}^{\prime}(u)=0$. But we already know $P_{E}^{\prime}(0)=0$. Hence $P_{E}^{\prime}(u) \neq 0$ implies $u \neq 0, C_{j}(u, t) \neq 0$.

Lemma 9.7. For $r<j \leq p$, we have

$$
D_{j}\left(u v^{h}, v, t\right)=\left(1+h^{2}\right)\left|c_{j}(t) w_{1}(u, t)\right|^{2} v^{2\left[L(E)-h+O_{y}\left(\delta_{j}\right)\right]}+\cdots .
$$

Hence if $P_{E}^{\prime}(u) \neq 0$, then $O_{v}\left(D_{j}\left(u v^{h}, v, t\right)\right)=2\left[L(E)-h+O_{y}\left(\delta_{j}\right)\right]$.

If $P_{E}(u) \neq 0$, then $O_{v}\left(D_{j}\left(u v^{h}, v, t\right)\right) \leq 2 L(E)$.

Proof. If $j>r$, then $h>O\left(\delta_{j}\right)$ and hence

$$
u v^{h}-\delta_{j}=-c_{j}(t) v^{O\left(\delta_{j}\right)}+\cdots, \quad c_{j}(0) \neq 0 .
$$

Then, as can be observed from (9.14), the leading term of $D_{j}$ is that of (9.15).

Next note that

$$
D_{j} \approx\left|\delta_{j} F_{X_{1}}\right|^{2}+\left|v F_{Y_{1}}+v \frac{\partial \delta_{j}}{\partial Y_{1}} F_{X_{1}}\right|^{2} \approx\left|\delta_{j} F_{X_{1}}\right|^{2}+\left|v F_{Y_{1}}\right|^{2} \geq\left|v F_{Y_{1}}\right|^{2},
$$

and that $O_{v}\left(v F_{Y_{1}}\left(u v^{r}, v, t\right)\right)=L(E)$ if $P_{E}(u) \neq 0$. This completes the proof.

The Newton dots of $F_{T_{j}}$ lie on or above the line $\mathcal{L}(E)$, hence the above lemmas imply that $A_{j} \circ S_{u v}, B_{j} \circ S_{u v}$ are real analytic at $(u, v, t)$ if $P_{E}^{\prime}(u) \neq 0,1 \leq j \leq p$.

Lemma 9.8. The denominator $D_{\mathcal{P}}:=\left|\hat{X}_{1}\right|^{2}+\cdots+\left|\hat{X}_{p}\right|^{2}$ of $\mathcal{P}_{k}$ has the form

$$
D_{\mathcal{P}}\left(u v^{h}, v, t\right)=c(u, t) v^{2 e}+\cdots, \quad e:=(r-1) h+\Sigma_{j>r} O\left(\delta_{j}\right),
$$

where $c(u, t)$ is a polynomial in $u, \bar{u}$.

If $\left(P_{E}(u), P_{E}^{\prime}(u)\right) \neq(0,0)$ then $c(u, t) \neq 0$.

Proof. For $1 \leq j \leq r$, let us write

$$
\mu_{j}(u, t):=u-\hat{c}_{j}(t), \quad \hat{\mu}_{j}(u, t):=\mu_{1} \cdots \mu_{j-1} \cdot \mu_{j+1} \cdots \mu_{r} .
$$

We can compute the $\hat{X}_{k}$ 's, $1 \leq k \leq p$, using the formula

$$
u v^{h}-\delta_{k}= \begin{cases}\left(u-\hat{c}_{k}(t)\right) v^{h}+\cdots, & \text { if } 1 \leq k \leq r, \\ -c_{k}(t) v^{O\left(\delta_{k}\right)}+\cdots, & \text { if } r<k \leq p,\end{cases}
$$

where $c_{k}(0) \neq 0$ for $r<k \leq p$. For example,

$$
\hat{X}_{1}=\left[(-1)^{p-r} c_{r+1}(t) \cdots c_{p}(t)\right]\left(u-\hat{c}_{2}(t)\right) \cdots\left(u-\hat{c}_{r}(t)\right) v^{e}+\cdots,
$$


where $e$ is defined in (9.16). The equations for the other $\hat{X}_{k}$ 's are similar.

Then (9.16) holds when we take

$$
c(u, t):=\left|c_{r+1}(t) \cdots c_{p}(t)\right|^{2} \cdot \sum_{j=1}^{r}\left|\hat{\mu}_{j}(u, t)\right|^{2} .
$$

If $c(u, t)=0$, then all $\hat{\mu}_{j}=0$, and there exist $i, j, 1 \leq i<j \leq r, u=\hat{c}_{i}(t)=\hat{c}_{j}(t)$. This implies, in particular, that $P_{E}^{\prime}(u)=0$.

We claim this also implies $P_{E}(u)=0$. Indeed, $\hat{c}_{i}(t)=\hat{c}_{j}(t)$ implies $O\left(\gamma_{i, \phi}-\gamma_{j, \phi}\right)>h / N$. If $P_{E}(u) \neq 0$, then $h / N=h\left(\gamma_{i, \phi}\right)=h\left(\gamma_{j, \phi}\right)$, hence $\gamma_{i, \phi}=\gamma_{j, \phi}$, a contradiction.

The $\mathcal{P}_{k}$ 's are bounded. Hence $\mathcal{P}_{k} \circ S_{u v}$ are real analytic at $(u, t)$ if $\left(P_{E}(u), P_{E}^{\prime}(u)\right) \neq(0,0)$.

Let $\mathcal{N}^{\prime}$ be an open neighborhood of the $u$-axis such that $(u, v) \in \mathcal{N}^{\prime}$ implies $\left(u v^{r}, v\right) \in \mathcal{N}$.

Answer to Question 9.5: Take $\left(u_{0}, v_{0}, t_{0}\right) \in \mathcal{N}^{\prime} \times I_{\mathbb{C}}$. If $\left(u_{0}, t_{0}\right)$ is not a root of $P_{E}^{\prime}(u)$, then $\vec{U}(u, v, t)$ is defined and real analytic in a neighborhood of $\left(u_{0}, v_{0}, t_{0}\right)$.

Lemma 9.9. Given $j, 1 \leq j \leq r$. For $(u, t)$ near $\left(\hat{c}_{j}(t), t\right)$,

$$
\left|\vec{U}(u, v, t)-\frac{\partial}{\partial t}\right| \lesssim\left|u-\hat{c}_{j}(t)\right|+|v| .
$$

Moreover, $\vec{U}(u, 0, t)$ is tangent to the $u$-coordinate space, and

$$
\left|\vec{U}(u, 0, t)-\frac{\partial}{\partial t}\right| \lesssim\left|u-\hat{c}_{j}(t)\right| .
$$

Proof. Let us first assume $j=1$, where $\hat{c}_{1}(t) \equiv 0$. The notations are simpler.

First, by (9.12),

$$
\left(d S_{u v}\right)^{-1}\left(\mathcal{P}_{1} \vec{V}_{1}\right)=-\mathcal{P}_{1} A_{1} u \frac{\partial}{\partial u}-\mathcal{P}_{1} B_{1}\left(-h u \frac{\partial}{\partial u}+v \frac{\partial}{\partial v}\right)+\mathcal{P}_{1} \frac{\partial}{\partial t},
$$

where $\mathcal{P}_{1} A_{1}, \mathcal{P}_{1} B_{1}$ are bounded.

For $k, 2 \leq k \leq p$, the identity

$$
\mathcal{P}_{k} X_{k} \frac{\partial}{\partial X_{k}}=\left\{\mathcal{P}_{k}^{1 / 2} \mathcal{P}_{1}^{1 / 2} X_{k}\left|X_{k}\right|^{-1}\right\}\left|X_{1}\right| \frac{\partial}{\partial X_{1}}
$$

and the Chain Rule (9.12) give

$$
\left(d S_{u v}\right)^{-1}\left(\mathcal{P}_{k} \vec{V}_{k}\right)=\tilde{A} u \frac{\partial}{\partial u}+\tilde{B} v \frac{\partial}{\partial v}+\frac{\partial}{\partial t},
$$

where $\tilde{A}, \tilde{B}$ are bounded.

Hence (9.17) is true for $j=1$.

Now assume $1<j \leq r$. This case is actually the same as the case $j=1$. For in (9.11), the roles of $\Gamma_{1}$ and $\Gamma_{j}$ are interchangeable. When $\Gamma_{j}$ replaces $\Gamma_{1}$, the leading coefficient $c_{k}(t)$ of $\delta_{k}$ in (9.9) is replaced by $c_{k}(t)-c_{j}(t)$. The same argument completes the proof of (9.17).

The $\frac{\partial}{\partial v}$ component vanishes when $v=0$. Hence $\vec{U}(u, 0, t)$ is tangent to the $u$-space. 
We are now ready to complete the proof of (3) in the case (a).

Recall that $\operatorname{Im}\left(\Gamma_{1}\right)$ is the vertical axis $X_{1}=0$, and $\rho_{X Y, \mathbb{C}}$ is defined by $X_{1}=u_{\rho} Y_{1}^{h}+\cdots$, which lifts to $\rho_{u v}: u=u_{\rho}+\cdots$.

We know $P_{E}^{\prime}\left(u_{\rho}\right) \neq 0$. Hence the arc $\rho_{u v}$ lies in the domain where $\vec{U}$ is analytic. The flow carries $\rho_{u v}$ to an analytic arc, at least for a sufficiently short time. Hence the flow of $\vec{v}$ carries $\rho_{X Y}$ to an analytic arc, at least for a short time. (Keep $|v|$ small, arcs short.)

This is actually so for all $t \in I_{\mathbb{C}}$, not just for a short time. Indeed, using Lemma 9.9, a well-known argument shows that a point on $\rho_{u v}$, following the flow, will never reach a point where $\vec{U}$ is not analytic, whence the trajectory is defined for all $t$.

The initial point $\left(u_{\rho}, 0,0\right)$ of $\rho_{u v}$ remains in the $u$-coordinate space for all time, because $\vec{U}$ is tangent to the $u$-space. By (9.18), it will never reach $\hat{c}_{j}(t)$.

Hence, downstairs, the geo-arc $\operatorname{Im}(\rho)$ is carried by the flow of $\overrightarrow{\mathcal{F}}$ real analytically, sweeping out a "geo-arc wing". This completes the proof of (3) in the case (a) when $h \in \mathbb{Z}^{+}$.

Suppose $h:=N_{1} / M_{1} \notin \mathbb{Z}^{+}$. We can make a further substitution $Y_{1} \mapsto Y_{1}^{M_{1}}$. The vector field lifts, retaining the same form, $h$ is magnified to $N_{1}$. The same argument applies.

Finally, consider case (b). This can be treated as a special case of (a), as follows.

In $\mathcal{N} \mathcal{P}\left(\phi, \gamma_{1, \phi}\right), E_{\text {top }}$ has left vertex $V_{l}=\left(0, q_{l}\right)$, since $h\left(\gamma_{1, \phi}\right)<\infty$. We may call $E:=\left(V_{l}, h\right)$ an "artificial vertex edge", of co-slope $h$, with Lojasiewicz exponent $L(E):=q_{l}$.

Let $V_{l}$ represent the term $c y^{q_{l}}, c \neq 0$. The associated polynomial is $P_{E}(z):=c$.

The above argument for the case (a) can then be repeated. It is actually easier. The lemmas remain true for the artificial vertex edge $E$, where now $P_{E}(u)=c \neq 0$.

This completes the proof of (3).

We then use the same idea to prove (4).

Take $\alpha_{* / f} \in \mathbb{C}_{* / f}$ and a canonical coordinate $\alpha_{\phi}(y)$. We can assume

$$
h\left(\alpha_{\phi}\right)=O_{y}\left(\alpha_{\phi}-\zeta_{1}\right) \geq O_{y}\left(\alpha_{\phi}-\zeta_{i}\right), \quad O_{y}\left(\alpha_{\phi}-\gamma_{1, \phi}\right) \geq O_{y}\left(\alpha_{\phi}-\gamma_{j, \phi}\right),
$$

where $\zeta_{i} \in Z(\phi), \gamma_{j, \phi} \in C\left(v_{a l}\right)$.

We now repeat the argument in (3) to show $\eta_{t}\left(\alpha_{* / f}\right)$ is well-defined. Let us write

$$
\alpha_{\phi}(y):=\zeta_{1}(y)+\left[u_{0} y^{h\left(\alpha_{\phi}\right)}+\cdots\right], \quad u_{0} \neq 0,
$$

where $\alpha_{* / f}$ is completely determined by $u_{0}$. (Terms in " $+\cdots "$ play no role.)

Here we have assumed that $h\left(\alpha_{\phi}\right)<\infty$. (If $\alpha_{\phi}=\zeta_{1}$, we can apply Corollary [5.6.)

Take an analytic arc $\rho$ such that $\pi_{*}\left(\rho_{\mathbb{C}}\right) \in \alpha_{* / f}$. As in (3), we lift $\rho$ to $\rho_{X Y}$, and analyze how $\rho_{X Y}$ is being carried by $\vec{v}(X, Y, T)$ in (8.9).

This time we use the coordinate system:

$$
X_{\zeta}:=X-\zeta_{1}\left(Y^{N}, T\right), \quad Y_{\zeta}:=Y, \quad T_{\zeta}:=T, \quad\left(y:=Y^{N}\right),
$$

where $N$ is divisible by $m_{\text {puis }}\left(\alpha_{\phi}\right)$ and $m_{\text {puis }}\left(\zeta_{i}\right)$.

The substitution $\left(X_{\zeta}, Y_{\zeta}\right):=\left(u v^{h}, v\right), h:=N h\left(\alpha_{\phi}\right)$, lifts $\rho_{X Y}$ to an analytic arc $\rho_{u v}$ in the $(u, v)$-space whose initial point is $\left(u_{0}, 0\right)$.

As in (3), $\vec{v}$ is lifted to $\vec{U} ; \rho_{u v}$ lies in the domain where $\vec{U}$ is real analytic. 
Hence, following the flow of $\vec{U}$ for time $t, \rho_{u v}$ reaches an analytic arc, denoted by $\rho_{u v, t}$, with initial point $\left(u_{t}, 0, t\right)$, where $u_{t}$ is real analytic in $t$.

An important observation is that $\left(u_{0}, 0\right)$, and hence also $\left(u_{t}, 0, t\right)$, depend only on $\alpha_{* / f}$, not on the choice of $\rho$. It follows that $\eta_{t}\left(\alpha_{* / f}\right)$ is well-defined.

(Note. Even if $\rho, \mu$ have the same complexification, $\operatorname{Im}\left(\rho_{\mathbb{C}}\right)=\operatorname{Im}\left(\mu_{\mathbb{C}}\right)$, the above argument does not prove that the geo-arcs $\operatorname{Im}\left(\eta_{t} \circ \rho\right), \operatorname{Im}\left(\eta_{t} \circ \mu\right)$ lie on a same curve-germ.

It merely shows that the curve-germs containing $\operatorname{Im}\left(\eta_{t} \circ \rho\right)$ and $\operatorname{Im}\left(\eta_{t} \circ \mu\right)$ are equivalent under $\sim_{F_{t}}$. This is the "Arakawa phenomenon": only the blurred point is well-defined.)

To complete the proof of (4), we need to know how to obtain $\mathcal{N} \mathcal{P}\left(f, \alpha_{\phi}\right)$ from $\mathcal{N} \mathcal{P}\left(f, \zeta_{1}\right)$.

If $h\left(\alpha_{\phi}\right)=\infty$, the two polygons are identical.

Assume $h\left(\alpha_{\phi}\right)<\infty\left(u_{0} \neq 0\right)$ in (9.20). Let $\left\{\left(m_{1}, q_{1}\right), \ldots,\left(m_{l}, q_{l}\right)\right\}$ denote the vertices of $\mathcal{N P}\left(f, \zeta_{1}\right)$, where, of course, $m_{l} \geq 1$.

Take $k(k \leq l)$ such that $\tan \theta_{k-1} \leq h\left(\alpha_{\phi}\right)<\tan \theta_{k}$.

Let $\left(0, q^{\prime}\right)$ be the left vertex of the edge $E\left(h\left(\alpha_{\phi}\right)\right)$. (If $\tan \theta_{k-1}<h\left(\alpha_{\phi}\right)<\tan \theta_{k}$, then $E\left(h\left(\alpha_{\phi}\right)\right)$ is a vertex edge. $)$ The vertices of $\mathcal{N} \mathcal{P}\left(f, \alpha_{\phi}\right)$ are

$$
\begin{cases}\left(m_{1}, q_{1}\right), \ldots,\left(m_{k-1}, q_{k-1}\right),\left(0, q^{\prime}\right), & \text { if } \tan \theta_{k-1}=h\left(\alpha_{\phi}\right), \\ \left(m_{1}, q_{1}\right), \ldots,\left(m_{l}, q_{l}\right),\left(0, q^{\prime}\right), & \text { if } \tan \theta_{k-1}<h\left(\alpha_{\phi}\right) .\end{cases}
$$

We can also obtain $\mathcal{N} \mathcal{P}\left(F_{t}, \eta_{t}\left(\alpha_{* / f}\right)\right)$ from $\mathcal{N} \mathcal{P}\left(F_{t}, \zeta_{1, t}\right)$ in the same way, since $u_{t} \neq 0$.

By Corollary 5.6, we know $\mathcal{N} \mathcal{P}\left(F_{t}, \zeta_{1, t}\right)=\mathcal{N} \mathcal{P}\left(f, \zeta_{1}\right)$. Hence $\mathcal{N} \mathcal{P}\left(F_{t}, \eta_{t}\left(\alpha_{* / f}\right)\right)=\mathcal{N} \mathcal{P}\left(f, \alpha_{\phi}\right)$. It follows that $h\left(\eta_{t}\left(\alpha_{* / f}\right)\right)$, $\chi_{\text {puis }}\left(\eta_{t}\left(\alpha_{* / t}\right)\right)$ are constants.

It remains to consider the contact order. From what we have proved,

$$
\mathcal{C}_{\text {ord }}\left(\alpha_{* / f}, \pi_{*}\left(\zeta_{i}\right)\right)=\mathcal{C}_{\text {ord }}\left(\eta_{t}\left(\alpha_{* / f}\right), \pi_{*}\left(\zeta_{i, t}\right)\right), \quad \zeta_{i} \in Z(\phi) .
$$

And, by Corollary 9.20 ,

$$
\mathcal{C}_{\text {ord }}\left(\pi_{*}\left(\zeta_{i, t}\right), \pi_{*}\left(\zeta_{j, t}\right)\right)=\mathcal{C}_{\text {ord }}\left(\pi_{*}\left(\zeta_{i}\right), \pi_{*}\left(\zeta_{j}\right)\right), \quad \zeta_{i}, \zeta_{j} \in Z(\phi)
$$

The same holds for $\beta_{* / f}$. It follows that $\mathcal{C}_{\text {ord }}\left(\eta_{t}\left(\alpha_{* / f}\right), \eta_{t}\left(\beta_{* / f}\right)\right)$ is independent of $t$. This completes the proof of (4).

The vector field $\overrightarrow{\mathcal{F}}$ is defined in such a way that $(5)$ is true.

Finally, suppose $\Phi$ is Morse stable. Then (3.3) is a consequence of (2.9). This completes the proof of the Equi-singular Deformation Theorem.

To prove the Truncation Theorem, note that $\mathcal{N} \mathcal{P}\left(f, \alpha_{\phi}\right)$ and $\mathcal{N} \mathcal{P}\left(u \cdot f, \alpha_{\phi}\right)$ have the same Newton dots, where $u(0,0) \neq 0$. (The dots in the interior of the polygons may be different.)

The monomial terms of $\mathcal{N} \mathcal{P}\left(u \cdot f, \alpha_{\phi}\right)$ are those of $\mathcal{N} \mathcal{P}\left(f, \alpha_{\phi}\right)$ multiplied by the same constant $u(0,0)$. Hence the critical points $C\left(v_{a l}\right)$ is unchanged when $f$ is multiplied by a unit, $F_{\text {root }}$ is obviously Morse stable. Now apply the Equi-singular Deformation Theorem. 


\section{Appendix 1. The Stability Theorem}

The Classical Morse Stability Theorem. A Morse stable family $\left\{p_{t}(x)\right\}$, as in Definition $\sqrt[5.4]{ }, \mathbb{K}=\mathbb{R}, \mathbb{C}$, or $\mathbb{F}$, admits a real-analytic trivialization. That is to say, there exist $t$-level preserving, real-analytic, homeomorphisms (deformations) $\mathcal{D}$ and $d$, such that

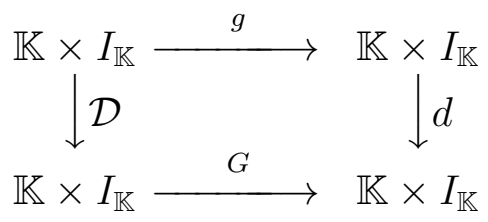

is commutative, where $d(0, t)=(0, t), g(x, t):=\left(p_{0}(x), t\right), G(x, t):=\left(p_{t}(x), t\right)$.

(In the case $\mathbb{K}=\mathbb{C}$, moduli can appear, we cannot ask $\mathcal{D}$, $d$ to be holomorphic.)

Proof. The critical value set of $G$ is, by definition,

$$
V_{\text {crit }}(G):=\left\{\left(v_{t}, t\right) \in \mathbb{K} \times I_{\mathbb{K}} \mid \exists c_{t} \in \mathbb{K}, p_{t}^{\prime}\left(c_{t}\right)=0, v_{t}=p_{t}\left(c_{t}\right)\right\},
$$

and $\pi: V_{\text {crit }}(G) \rightarrow I_{\mathbb{K}}$ is a fibration, $\{0\} \times I_{\mathbb{K}}$ is either disjoint from, or contained in, $V_{\text {crit }}(G)$.

Let us first consider the case $\mathbb{K}=\mathbb{R}$, which exposes the main ideas. Take a vector field

$$
\vec{v}(x, t):=a(x, t) \frac{\partial}{\partial x}+\frac{\partial}{\partial t}, \quad a(x, t) \text { analytic }
$$

which is defined on, and tangent to, $V_{\text {crit }}(G) \cup\left(\{0\} \times I_{\mathbb{R}}\right)$.

Then, using Cartan's Theorem B, or the Lagrange Interpolation Formula, we can extend $a(x, t)$ to a real analytic function defined for $(x, t) \in \mathbb{R} \times I_{\mathbb{R}}$.

In other words, $\vec{v}(x, t)$ is now defined and real analytic on $\mathbb{R} \times I_{\mathbb{R}}$, tangent to $V_{\text {crit }}(G)$ and $\{0\} \times I_{\mathbb{R}}$. Integrating this vector field gives an analytic deformation

$$
d: \mathbb{R} \times I_{\mathbb{R}} \rightarrow \mathbb{R} \times I_{\mathbb{R}}, \quad(x, t) \mapsto\left(x_{t}, t\right),
$$

such that $d\left(V_{\text {crit }}(g)\right)=V_{\text {crit }}(G)$, and $\{0\} \times I_{\mathbb{R}}$ is fixed.

Using $d$ as an identification, we assume $V_{\text {crit }}(g)=V_{\text {crit }}(G)$ ("straightening up" $V_{\text {crit }}(G)$ ).

Take $c \in C\left(p_{0}\right), p_{0}(c):=v$. Then $c$ admits a unique continuous deformation $c_{t}$ in $C\left(p_{t}\right)$, $p_{t}\left(c_{t}\right)=v, c_{0}=c$. As $m_{c r i t}\left(c_{t}\right)$ is constant, $c_{t}$ is necessarily real analytic. Hence

$$
p_{t}(x)-v=\text { unit } \cdot\left(x-c_{t}\right)^{m_{\text {crit }}(c)+1},(x, t) \text { near }\left(c_{t}, t\right) \text {, }
$$

Then $\frac{\partial}{\partial t}$ lifts to a unique real analytic vector field in $\mathbb{R} \times I_{\mathbb{R}}$ which generates $\mathcal{D}$.

For the case $\mathbb{K}=\mathbb{C}$, we still have $d$, which is (merely) real analytic, and also (10.1), where $c_{t}$ is holomorphic in $t$. Thus $\frac{\partial}{\partial t}$ admits a local real analytic lifting. By Cartan's Theorem B, a real analytic global lifting exists. This completes the proof. 


\section{Appendix 2. Tree Models}

The tree-model ([13]) is best explained by an example. Take $\phi(\xi)=\left(\xi^{2}-y^{3}\right)^{2}-4 \xi y^{5}$. The Puiseux roots are

$$
\zeta_{i}(y)= \pm y^{3 / 2} \pm y^{7 / 4}+\cdots, \quad 1 \leq i \leq 4 .
$$

The tree-model is shown in Fig. 3. Tracing upward from the tree root to a tip along solid line segments amounts to identifying a Puiseux root.

There are three bars, of height $3 / 2,7 / 4,7 / 4$ respectively. Each is indicated by a horizontal line segment (whence the name); the associated polynomial has at least two distinct roots.

At these heights, the $\zeta_{i}$ 's split away from each other. Bars without this property are not indicated; they correspond to the vertex edges, Fig.4.

The three critical points (polars) $\gamma_{j}$ are indicated by dashed lines; their positions relative to the $\zeta_{i}$ are justified by Theorem [5.3, exposing the contact orders.

The Newton Polygon $\mathcal{N} \mathcal{P}\left(\phi, \zeta_{i}\right)$ is shown in Fig.4. The dotted segments $e_{1}, e_{2}, e_{3}$ indicate vertex edges, where

$$
h\left(e_{1}\right)<3 / 2<h\left(e_{2}\right)<7 / 4<h\left(e_{3}\right)<\infty .
$$

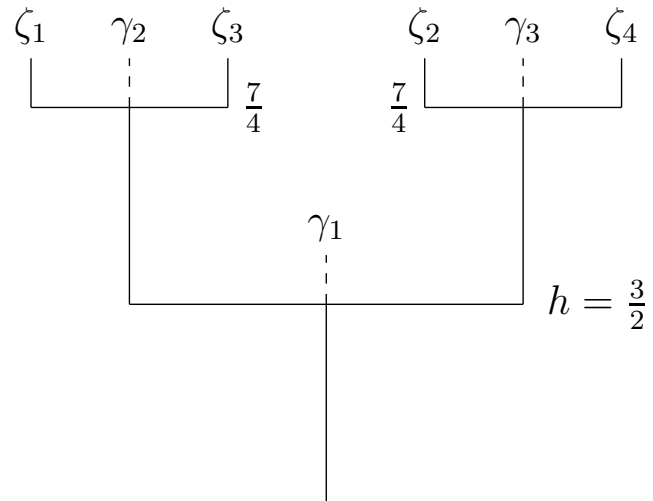

Figure 3. Tree Model $\mathbb{M}_{1, \phi}$

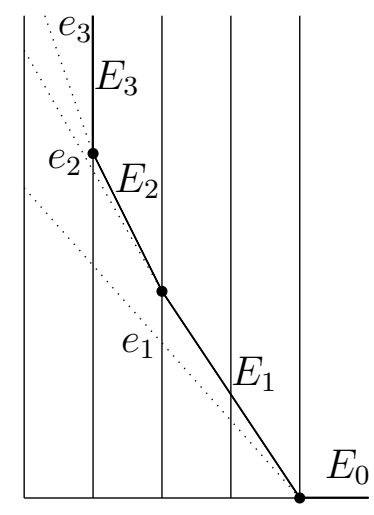

FigURE $4 . \mathcal{N} \mathcal{P}\left(\phi, \zeta_{i}\right)$

\section{REFERENCES}

1. Abderrahmane J, Ould M., Newton Polygon and Trivialisation of Families, J. Math. Soc. Japan, 54, 513-550 (2002).

2. K. Bekka and S. Koike, The Kuo condition, an inequality of Thom's type and $(C)$-regularity, Topology, 37 (1998), 45-62.

3. E. Bierstone and P. D. Milman, Arc-analytic functions, Invent. math., 101 (1990), 411-424.

4. E. Bierstone, P. Milman Semianalytic and subanalytic sets, Inst. Hautes tudes Sci. Publ. Math. No. 67, $1988,5-42$.

5. J. Bochnak, S. Lojasiwicz A Converse of the Kuiper-Kuo Theorem, in Proc. Liverpool Singularities Symposium, I, (ed. C.T.C. Wall), 1971, Lecture Notes Math., 209, Springer-Verlag.

6. E. Brieskorn, H. Knörrer, Plane Algebraic Curves, Birkhäuser Verlag, 1986.

7. Charles Favre, Mattias Jonsson, Valuative analysis of planar plurisubharmonic functions, Invent. Math. 162 (2005), No. 2, pp 271-311. 
8. T. Fukui and L. Paunescu, Modified Analytic Trivialization for Weighted Homogeneous Function Germs, J. Math. Soc. Japan, vol. 52, No. 2, (2000), pp 433-446.

9. H. Hancock, Theory of Maxima and Minima, Dover Publications, Inc. New York 1960.

10. Theo de Jong, Gerhard Pfister, Local analytic geometry. Basic theory and applications, Advanced Lectures in Mathematics. Friedr. Vieweg \& Sohn, Braunschweig, 2000.

11. S. Koike, Notes on $C^{0}$-sufficiency of quasijets, J. Math. Soc. Japan, 42 (1990), 265-275.

12. T.-C. Kuo, On $C^{0}$-sufficiency of jets of potential function, Topology 8, p.157-171 (1969).

13. T.-C. Kuo and Y.C. Lu, On analytic function germs of two complex variables, Topology, 15 (1977), 299-310.

14. T.-C. Kuo and A. Parusiński, Newton Polygon relative to an arc, in Real and Complex Singularities (São Carlos, 1998), Chapman \& Hall Res. Notes Math., 412, 2000, 76-93.

15. T.-C. Kuo and L. Paunescu, Equisingularity in $R^{2}$ as Morse stability in infinitesimal calculus, (Communicated by H. Hironaka) Proc. Japan Acad. Ser. A, Math. Sci. Volume 81, Number 6 (2005), 115-120.

16. J. Milnor, Morse Theory, Annals of Math. Studies. 51, Princeton Univ. Press 1983.

17. J. Milnor, Singular Points of Complex Hypersurfaces, Annals of Math. Studies. 61, Princeton Univ. Press 1968.

18. L. Paunescu, V-sufficiency from the weighted point of view, J. Math. Soc. Japan, vol. 46, No. 2, (1994), pp $345-354$.

19. L. Paunescu, A Weighted Version of the Kuiper-Kuo-Bochnak-Lojasiewicz Theorem, J. of Algebraic Geometry, volume 2, number 1, January (1993), pp 69-79.

20. F. Pham, Deformations equisingularitiés des ideaux jacobiens de courbes planes, in Proc. Liverpool Singularities Sym., II, (ed. C.T.C. Wall), 1971, 218-233, Lecture Notes Math., 209, Springer-Verlag.

21. van der Waerden, Einführung in die Algebraische Geometrie, Zweite Auflage. Die Grundlehren der Mathematischen Wissenschaften, Band 51. Springer-Verlag, Berlin-New York, 1973.

22. R. J. Walker, Algebraic Curves, Springer-Verlag, 1972.

23. H. Whitney, Complex Analytic Varieties, Addison-Wesley, Reading, Mass., 1972.

24. O. Zariski, Algebraic Surfaces, Ergebnisse der Mathematik und ihrer Grenzgebiete, Band 61. SpringerVerlag, New York-Heidelberg, 1971.

25. O. Zariski, Studies in equisingularity I, Amer J. Math. 2, 87, (1965), 507-536.

School of Mathematics and Statistics, University of Sydney, Sydney, NSW, 2006, AusTRALIA

E-mail address: tck@maths.usyd.edu.au; laurent@maths.usyd.edu.au 